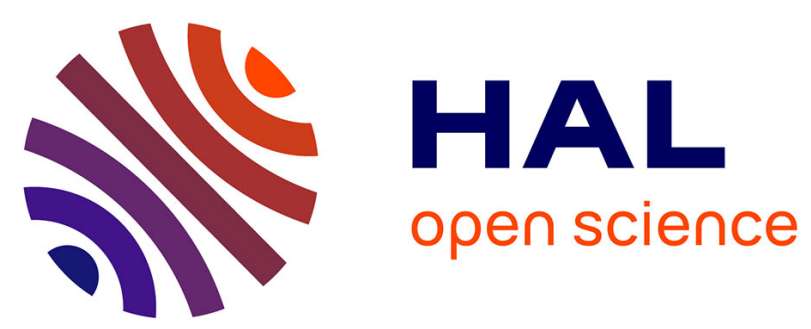

\title{
Multi-speed solitary waves of nonlinear Schrödinger systems: theoretical and numerical analysis
} Fanny Delebecque, Stefan Le Coz, Rada-Maria Weishäupl

\section{To cite this version:}

Fanny Delebecque, Stefan Le Coz, Rada-Maria Weishäupl. Multi-speed solitary waves of nonlinear Schrödinger systems: theoretical and numerical analysis . Communications in Mathematical Sciences, 2016, 14 (6), pp.1599-1624. 10.4310/CMS.2016.v14.n6.a7 . hal-01140207v3

\section{HAL Id: hal-01140207 \\ https://hal.science/hal-01140207v3}

Submitted on 21 May 2015

HAL is a multi-disciplinary open access archive for the deposit and dissemination of scientific research documents, whether they are published or not. The documents may come from teaching and research institutions in France or abroad, or from public or private research centers.
L'archive ouverte pluridisciplinaire HAL, est destinée au dépôt et à la diffusion de documents scientifiques de niveau recherche, publiés ou non, émanant des établissements d'enseignement et de recherche français ou étrangers, des laboratoires publics ou privés. 


\title{
MULTI-SPEED SOLITARY WAVES OF NONLINEAR SCHRÖDINGER SYSTEMS: THEORETICAL AND NUMERICAL ANALYSIS
}

\author{
FANNY DELEBECQUE, STEFAN LE COZ, AND RADA M. WEISHÄUPL
}

\begin{abstract}
We consider a system of coupled nonlinear Schrödinger equations in one space dimension. First, we prove the existence of multi-speed solitary waves, i.e solutions to the system with each component behaving at large times as a solitary wave. Then, we investigate numerically the interaction of two solitary waves supported each on one component. Among the possible outcomes, we find elastic and inelastic interactions, collision with mass extraction and reflexion.
\end{abstract}

\section{Contents}

\begin{tabular}{|lr|}
\hline 1. Introduction & 2 \\
\hline 1.1. The theoretical result & 3 \\
\hline 1.2. The numerical experiments & 4 \\
\hline 2. Existence of multi-speed solitary waves & 5 \\
\hline 3. Uniform estimates & 6 \\
\hline 3.1. The bootstrap argument & 6 \\
\hline 3.2. Modulation & 7 \\
\hline 3.3. Energy estimates and coercivity & 8 \\
\hline 3.4. Almost-conservation of the localized momentum & 12 \\
\hline 3.5. Control of the modulation parameters & 14 \\
\hline 3.6. Conclusion & 14 \\
\hline $4 . \quad$ Numerical schemes & 15 \\
\hline 4.1. The time-splitting spectral method & 15 \\
\hline 4.2. The normalized gradient flow & 16 \\
\hline $5 . \quad$ Numerical experiments & 16 \\
\hline $5.1 . \quad$ Purely elastic interaction & 17 \\
\hline $5.2 . \quad$ Symmetric collision & 18 \\
\hline $5.3 . \quad$ Dispersive inelastic interaction & 19 \\
\hline $5.4 . \quad$ Reflexion & 20 \\
\hline Appendix A. Proof of the modulation lemma & 21 \\
\hline References & 24
\end{tabular}

Date: May 27, 2015.

2010 Mathematics Subject Classification. 35Q55(35C08,35Q51,37K40).

Key words and phrases. solitons, solitary waves, nonlinear Schrödinger systems.

The work of F. D. is partially supported by PHC AMADEUS 2014 31471ZK.

The work of S. L. C. is partially supported by ANR-11-LABX-0040-CIMI within the program ANR-11-IDEX-0002-02, ANR-14-CE25-0009-01 and PHC AMADEUS 2014 31471ZK.

The work of R.M.W. is supported by the FWF Hertha-Firnberg Program, Grant T402-N13 and Austrian-French Project WTZ-Amadée FR 18/2014. 


\section{INTRODUCTION}

We consider the following nonlinear Schrödinger system:

$$
\left\{\begin{array}{l}
i \partial_{t} u_{1}+\partial_{x x} u_{1}+\mu_{1}\left|u_{1}\right|^{2} u_{1}+\beta\left|u_{2}\right|^{2} u_{1}=0 \\
i \partial_{t} u_{2}+\partial_{x x} u_{2}+\mu_{2}\left|u_{2}\right|^{2} u_{2}+\beta\left|u_{1}\right|^{2} u_{2}=0
\end{array}\right.
$$

where for $j=1,2$ we have $u_{j}: \mathbb{R} \times \mathbb{R} \rightarrow \mathbb{C}, \mu_{j}>0$, and $\beta \in \mathbb{R} \backslash\{0\}$.

When $\mu_{1}=\mu_{2}=\beta$, system (NLS), also called Manakov system has been introduced by Manakov (see 21] for example) as an asymptotic model for the propagation of electric fields in waveguides. In this particular case, it is to be noticed that the usual roles of $x$ and $t$ are inverted to study the evolution of the electrical field along the propagation axis.

It has also been used later on to model the evolution of light in optical fiber links. One of the main limiting effects of transmission in optical fiber links is due to the polarization mode dispersion (PMD). It can be explained by the birefringence effect, i.e the fact that the electric field is a vector field and that the refraction index of the medium depends on the polarization state (see e.g [1, 2]). The evolution of two polarized modes of an electrical field in a birefringent optical fiber link can indeed be modeled by (NLS) in the case where $\mu_{1}=\mu_{2}$ and $\beta$ measures the strength of the cross phase modulation which depends of the fiber (see 21]). Randomly varying birefringence is studied adding random coefficients in both nonlinearity and coupling terms of (NLS) (see for example 15])

In higher dimensions, systems of nonlinear coupled Schrödinger equations appears in various physical situations such as the modeling of the interaction of two Bose-Einstein condensates in different spin states.

Systems of type (NLS) have also been studied from the mathematical point of view. When $\mu_{1}=\mu_{2}=\beta$, in dimension 1 , the system (NLS) has the particularity to be completely integrable. Hence explicit calculations of solutions are possible and one can exhibit a variety of "truly" nonlinear solutions like solitons, multi-solitons or breathers (see e.g. the book 1]). The integrability property is however not robust, and the slightest change in the parameters $\mu_{1}, \mu_{2}$ and $\beta$ destroys it. Many works (see, among many others, [3, 8, 14, 29]) have been devoted to the study of the stationary version of (NLS)

$$
\left\{\begin{array}{l}
\partial_{x x} \phi_{1}+\mu_{1}\left|\phi_{1}\right|^{2} \phi_{1}+\beta\left|\phi_{2}\right|^{2} \phi_{1}=\omega_{1} \phi_{1}, \\
\partial_{x x} \phi_{2}+\mu_{2}\left|\phi_{2}\right|^{2} \phi_{2}+\beta\left|\phi_{1}\right|^{2} \phi_{2}=\omega_{2} \phi_{2},
\end{array}\right.
$$

that one obtains when looking for standing waves solutions

$$
\left(u_{1}, u_{2}\right)(t, x)=\left(e^{i \omega_{1} t} \phi_{1}(x), e^{i \omega_{2} t} \phi_{2}(x)\right) .
$$

When standing waves exist, it is natural to study their stability and again many works have been devoted to this problem (see, again among many others, [16, 20, 26. 28]). Existence and stability of standing waves are often proved using variational techniques. The analysis of (11) through variational techniques is very subtle and the introduction of new ideas is necessary to understand the full picture (see [27]). Note that (NLS) is Galilean-invariant. Hence a Galilean transform modifies a standing wave into a solitary wave traveling at some non-zero speed.

Our goal in this paper is to provide a new point of view on the study of this system. We aim at understanding better the behavior in large times of solutions 
starting at initial time as two scalar solitary waves carried by the two different components. We will use a mixture of theoretical and numerical tools, a combination seldom seen when dealing with this kind of problems.

First, we propose to push further a study initiated in [17 on the multi-speed solitary waves of system (NLS) and followed up for a different nonlinearity in 30. A multi-speed solitary wave is a solution of (NLS) which behaves at large time as two solitary waves. Here and as in [17, we restrict ourselves to the case where the composing solitary waves are each carried on only one component of the system. In other words, taken independently, each component behaves as a scalar solitary wave at large time. Our first aim is to remove the high speed assumption under which the main result in [17] was proved. We therefore consider the system (NLS) in dimension 1 and benefit from the fact that scalar solitary waves are in that case orbitally stable (see e.g [11]).

Our next aim is to investigate further the properties of multi-speed solitary waves solutions when they are crossing at positive time. Our theoretical result (Theorem 1) indeed only guarantees existence of multi-speed solitary wave solutions to (NLS) when no interaction can occur at large time between the composing waves. But what happens when two solitary waves carried by different components collide? Due to the possible complexity of the phenomenon and the lack of appropriate theoretical tools to study it, we proceed the following numerical experiment. We take as initial data solitons on each components, both away from 0 but facing each other for the direction of propagation. Among the possible outcomes, we find elastic and inelastic interactions, interaction with mass exctraction, and reflexion.

1.1. The theoretical result. Before stating our main theoretical result, let us give a few preliminaries.

Let $Q_{\omega} \in H^{1}(\mathbb{R})$ be the unique positive radial ground state solution to

$$
-\partial_{x x} Q_{\omega}+\omega Q_{\omega}-\left|Q_{\omega}\right|^{2} Q_{\omega}=0, \quad Q_{\omega}>0, \quad Q_{\omega} \in H_{\mathrm{rad}}^{1}(\mathbb{R}) .
$$

From simple calculations we note that the following scaling occurs:

$$
Q_{\omega}(x):=\sqrt{\omega} Q_{1}(\sqrt{\omega} x) .
$$

For $j=1,2$, consider $\omega_{j}>0, \gamma_{j} \in \mathbb{R}, x_{j}, v_{j} \in \mathbb{R}$ and define

$$
R_{j}(t, x)=e^{i\left(\omega_{j} t-\frac{1}{4}\left|v_{j}\right|^{2} t+\frac{1}{2} v_{j} \cdot x+\gamma_{j}\right)} \sqrt{\frac{1}{\mu_{j}}} Q_{\omega_{j}}\left(x-v_{j} t-x_{j}\right) .
$$

The function $R_{j}$ is a solitary wave solution to

$$
i \partial_{t} u+\partial_{x x} u+\mu_{j}|u|^{2} u=0 .
$$

In this paper, we want to investigate the existence of solutions to (NLS) where each component behaves like a solitary wave $R_{j}$ solution to the scalar equation (5). Our main theoretical result is the following.

Theorem 1. Let $\mu_{1}, \mu_{2}>0$ and $\beta \in \mathbb{R} \backslash\{0\}$. For $j=1,2$, take $v_{j}, x_{j}, \gamma_{j} \in \mathbb{R}$, $\omega_{j}>0$ and consider the ground state profile $Q_{\omega_{j}}$ solution to (2) and the soliton $R_{j}$ defined in (4). Then, there exist $C>0, T_{0}>0$ and $\left(u_{1}, u_{2}\right)$ solution to (NLS) on the time interval $\left[T_{0},+\infty\right)$ such that for all $t \in\left[T_{0},+\infty\right)$, we have

$$
\left\|\left(\begin{array}{l}
u_{1}(t) \\
u_{2}(t)
\end{array}\right)-\left(\begin{array}{l}
R_{1}(t) \\
R_{2}(t)
\end{array}\right)\right\|_{H^{1} \times H^{1}} \leqslant C e^{-\sqrt{\omega_{*}} v_{*} t},
$$


where $\omega_{*}=\frac{1}{2304} \min \left\{\omega_{1}, \omega_{2}\right\}$ and $v_{*}=\left|v_{1}-v_{2}\right|$.

Remark 1. Compare to [17, Theorem 1], the main differences are the following. Our result is valid for any speeds, whereas the one in [17] required a high speed assumption. We restrict ourselves to dimension 1 to have stable solitons (in [17, any dimension was allowed). The overall proof strategy is similar, but in our case we need to perform several technical refinements which include in particular working with localized momenta and modulated waves.

In addition, we are introducing the technical artefact consisting into introducing arbitrary constants in the definition (15) of the global action. This is a new feature for this type of analysis, which is quite surprising as usually such a flexibility is not allowed by the algebra of the problem.

The scheme of the proof is inspired by the one developed for the study of multisolitons in scalar nonlinear Schrödinger equations in [12, 13, 23, 25] (see also 9] for a similar approach applied to Klein-Gordon equations). It consists in solving (NLS) backward in time, taking as final data a couple of solitary waves $\left(R_{1}\left(T^{n}\right), R_{2}\left(T^{n}\right)\right)$, for an increasing sequence of times $T^{n} \rightarrow+\infty$. Thus we get a sequence $\left(u_{1}^{n}, u_{2}^{n}\right)$ of solutions to (NLS) on a time interval $\left(-\infty, T^{n}\right]$ such that $\left(u_{1}^{n}\left(T^{n}\right), u_{2}^{n}\left(T^{n}\right)\right)=$ $\left(R_{1}\left(T^{n}\right), R_{2}\left(T^{n}\right)\right)$. We then have to prove the existence of a time $T_{0}$, independent of $n$ such that for $n$ large enough, $\left(u_{1}^{n}, u_{2}^{n}\right)$ is close to $\left(R_{1}, R_{2}\right)$ on $\left[T_{0}, T^{n}\right]$. The key tools at hand to prove Theorem 1 are

- uniform in $n$ estimates

$$
\forall t \in\left[T_{0}, T^{n}\right],\left\|\left(\begin{array}{l}
u_{1}^{n}(t) \\
u_{2}^{n}(t)
\end{array}\right)-\left(\begin{array}{l}
R_{1}(t) \\
R_{2}(t)
\end{array}\right)\right\|_{H^{1} \times H^{1}} \leqslant C e^{-\sqrt{\omega_{*}} v_{*} t}
$$

- a compactness argument that gives the existence of $\left(u_{1}^{0}, u_{2}^{0}\right) \in H^{1}(\mathbb{R}) \times$ $H^{1}(\mathbb{R})$ such that $\left(u_{1}^{n}, u_{2}^{n}\right)$ converges strongly in $H^{s}(\mathbb{R})(s \in[0,1))$ towards $\left(u_{1}^{0}, u_{2}^{0}\right)$.

Remark 2. The method used to obtain Theorem 1 is a powerful tool to obtain sharp existence results for multi-solitons composed of ground states. Another approach relying on a fixed point argument has been developed for nonlinear Schrödinger equations in [18, 19]. This approach is very flexible and allows to prove existence of solutions more complicated than the ones in Theorem 1 like infinite trains of solitons or multi-kinks. The main drawback is that it always requires a large speed assumption.

We also have tested numerically if the multi-speed solitary wave configuration was stable provided the starting waves are well-ordered and well-separated. In other words, we took the interaction to be small at the origin and the composing waves going away from each other. With such a well-prepared initial configuration, we remain close to a similar configuration in large time. This suggests that the multi-speed solitary waves are stable (no matter the coupling parameter). Note that this is expected due to the fact that each wave taken individually is stable. We have however no theoretical mean to verify this conjecture. Similar difficulties arise in the analysis of the stability for multi-solitons in nonlinear scalar Schrödinger equations (see e.g. 24]).

1.2. The numerical experiments. We solve the system (NLS) in one dimension by adapting the time-splitting spectral method described in 7 . This method 
is unconditionally stable, time reversible, of spectral-order accuracy in space and second-order accuracy in time, and it conserves the discrete total mass [5]. One can refer to [4] for other possible schemes and their properties.

We will also compute the real valued ground state (minimizer of the energy on fixed $L^{2}$ mass constraints) of the system (1) using a normalized gradient flow approach. This will be used to make the comparison between the outcome of the interaction between two solitary waves and a solitary wave with profile $\left(\phi_{1}, \phi_{2}\right)$.

As already mentioned, the experiment consists in taking as initial data the initial data of two solitary waves facing each other, each on one component.

We considered four cases, the first one being the integrable case, where we expect the solitons after the interaction to move with the same velocity and amplitude. Apart when $\mu_{1}=\mu_{2}=\beta$, the system is not integrable, hence we do not expect pure elastic interaction between solitons. However, there are still regimes where we expect the outcome of interaction between solitons to be also a multi-speeds solitary wave, different from the input at two level: first, there are modifications in the speeds and amplitudes of the composing solitons. Second, there is a loss of a bit of energy, mass and momentum into a small dispersive remainder. In certain cases, we have been able to identify the profiles of the outcome of the interactions as ground states of the stationary system (11).

The rest of this paper is organized as follows. In Section 2, we prove Theorem 1 assuming uniform estimates. In Section 3, we prove the uniform estimates. The numerical methods are described in Section 4 , and the numerical experiments are presented in Section 5. Appendix $\mathrm{A}$ contains the proof of a modulation result.

\section{Existence OF MUlti-SPEED SOlitARY WAVES}

This short section is devoted to the proof of Theorem 1, assuming uniform estimates proved in the next section.

In this section and in the next one, we assume that $\mu_{1}, \mu_{2}>0$ and $\beta \in \backslash\{0\}$ are fixed constants and that we are given for $j=1,2$ soliton parameters $\omega_{j}, v_{j}, x_{j}, \gamma_{j} \in$ $\mathbb{R}$. Denote by $Q_{\omega_{j}}$ and $R_{j}$ the corresponding profile and soliton.

We make the assumption that

$$
0<v_{1}=-v_{2} .
$$

Since (NLS) is Galilean invariant, this assumption can be done without loss of generality. This will simplify calculations later on.

Note that it follows from classical arguments (see [10]) that the Cauchy problem for (NLS) is globally well-posed in the energy space $H^{1}(\mathbb{R}) \times H^{1}(\mathbb{R})$ and also in $L^{2}(\mathbb{R}) \times L^{2}(\mathbb{R})$. In particular, for any initial data $\left(u_{1}^{0}, u_{2}^{0}\right) \in H^{1}(\mathbb{R}) \times H^{1}(\mathbb{R})$ there exists a unique global solution $\left(u_{1}, u_{2}\right)$ of (NLS) in $\mathcal{C}\left(\mathbb{R}, H^{1}(\mathbb{R}) \times H^{1}(\mathbb{R})\right) \cap$ $\mathcal{C}^{1}\left(\mathbb{R}, H^{-1}(\mathbb{R}) \times H^{-1}(\mathbb{R})\right)$.

Let $\left(T^{n}\right)$ be an increasing sequence of times such that $T^{n} \rightarrow+\infty$ as $n \rightarrow$ $+\infty$. Let $\left(u_{1}^{n}, u_{2}^{n}\right)$ be the sequence of solutions to (NLS) defined by solving (NLS) backward on $\left(-\infty, T^{n}\right]$ with final data $\left(u_{1}^{n}, u_{2}^{n}\right)\left(T^{n}\right)=\left(R_{1}, R_{2}\right)\left(T^{n}\right)$. The proof of Theorem 1 then relies on the following two ingredients.

First, we have uniform estimates on the distance between the sequence $\left(u_{1}^{n}, u_{2}^{n}\right)$ and the multi-speed solitary wave profile $\left(R_{1}, R_{2}\right)$. 
Proposition 3 (Uniform estimates). There exist $T_{0}>0, n_{0} \in \mathbb{N}$ such that, for all $n \geqslant n_{0}$ and for all $t \in\left[T_{0}, T^{n}\right]$ we have

$$
\left\|\left(\begin{array}{l}
u_{1}^{n} \\
u_{2}^{n}
\end{array}\right)(t)-\left(\begin{array}{l}
R_{1} \\
R_{2}
\end{array}\right)(t)\right\|_{H^{1} \times H^{1}} \leqslant e^{-\sqrt{\omega_{*}} v_{*} t},
$$

where, as in Theorem 1, $\omega_{*}=\frac{1}{2304} \min \left\{\omega_{1}, \omega_{2}\right\}$ and $v_{*}=\left|v_{1}-v_{2}\right|$.

The proof of Proposition 3 is rather involved and we postpone it to Section 3

The next ingredient is a compactness result on the initial data $\left(u_{1}^{n}, u_{2}^{n}\right)\left(T_{0}\right)$. This result was already present in this form in [17] and we recall it without proof.

Proposition 4 (Compactness). There exists $\left(u_{1}^{0}, u_{2}^{0}\right) \in H^{1}(\mathbb{R}) \times H^{1}(\mathbb{R})$ such that, up to a subsequence, $\left(u_{1}^{n}, u_{2}^{n}\right)\left(T_{0}\right)$ converges strongly towards $\left(u_{1}^{0}, u_{2}^{0}\right)$ in $H^{s}(\mathbb{R}) \times$ $H^{s}(\mathbb{R})$ for all $s \in[0,1)$.

With these two ingredients in hand, we can now conclude the proof of Theorem 1

Proof of Theorem 1. Let $\left(u_{1}, u_{2}\right)$ be the solution on $\mathbb{R}$ of the Cauchy problem (NLS) with initial data $\left(u_{1}^{0}, u_{2}^{0}\right)$ at $t=T_{0}$. By $H^{1}(\mathbb{R}) \times H^{1}(\mathbb{R})$ boundedness and local well-posedness of the Cauchy problem in $H^{s}(\mathbb{R}) \times H^{s}(\mathbb{R})$ for all $s \in[0,1)$, we have weak convergence in $H^{1}(\mathbb{R}) \times H^{1}(\mathbb{R})$ of $\left(u_{1}^{n}, u_{2}^{n}\right)(t)$ towards $\left(u_{1}, u_{2}\right)(t)$ for any $t \in \mathbb{R}$. Combined with the uniform estimates of Proposition 3 this implies for all $t \in\left[T_{0},+\infty\right)$ that

$\left\|\left(\begin{array}{l}u_{1} \\ u_{2}\end{array}\right)(t)-\left(\begin{array}{l}R_{1} \\ R_{2}\end{array}\right)(t)\right\|_{H^{1} \times H^{1}} \leqslant \liminf _{n \rightarrow+\infty}\left\|\left(\begin{array}{c}u_{1}^{n} \\ u_{2}^{n}\end{array}\right)(t)-\left(\begin{array}{l}R_{1} \\ R_{2}\end{array}\right)(t)\right\|_{H^{1} \times H^{1}} \leqslant C e^{-\sqrt{\omega_{*}} v_{*} t}$.

This concludes the proof of Theorem 1

\section{UNIFORM ESTIMATES}

This section is devoted to the proof of Proposition 3, In all this section, $T^{n}$ and $\left(u_{1}^{n}, u_{2}^{n}\right)$ are given as in the beginning of Section 2

3.1. The bootstrap argument. We first reduce the proof of Proposition 3 to the proof of the following bootstrap result.

Proposition 5 (Bootstrap argument). There exist $T_{0}>0$ and $n_{0} \in \mathbb{N}$ such that for all $n \geqslant n_{0}$ and for any $t_{0} \in\left[T_{0}, T^{n}\right]$ the following property is satisfied. If for all $t \in\left[t_{0}, T^{n}\right]$ we have

$$
\left\|\left(\begin{array}{l}
u_{1}^{n} \\
u_{2}^{n}
\end{array}\right)(t)-\left(\begin{array}{l}
R_{1} \\
R_{2}
\end{array}\right)(t)\right\|_{H^{1} \times H^{1}} \leqslant e^{-\sqrt{\omega_{*}} v_{*} t},
$$

then for all $t \in\left[t_{0}, T^{n}\right]$ we have

$$
\left\|\left(\begin{array}{l}
u_{1}^{n} \\
u_{2}^{n}
\end{array}\right)(t)-\left(\begin{array}{l}
R_{1} \\
R_{2}
\end{array}\right)(t)\right\|_{H^{1} \times H^{1}} \leqslant \frac{1}{2} e^{-\sqrt{\omega_{*}} v_{*} t} .
$$

The proof of Proposition 5 will occupy us for most of the rest of this section. We divided it into several steps. We first perform a geometrical decomposition of the sequence $\left(u_{1}^{n}, u_{2}^{n}\right)$ onto the manifold of multi-speed solitary waves in order to obtain orthogonality conditions. We then introduce an action-like functional, which turns out to be coercive due to our orthogonality conditions. This functional is not a conserved quantity, but since it is made with localized conservations laws it is 
almost conserved. Using that property and a control on the geometrical modulation parameters, we are able to conclude the proof of Proposition 5

Before going on with the details of the proof of Proposition [ 5 let us show how it implies Proposition 3

Proof of Proposition [3. Since we have $\left(u_{1}^{n}, u_{2}^{n}\right)\left(T^{n}\right)=\left(R_{1}^{n}, R_{2}^{n}\right)\left(T^{n}\right)$ at the final time $T^{n}$, by continuity there exists a minimal time $t_{0}$ such that for all $t \in\left[t_{0}, T^{n}\right]$ we have

$$
\left\|\left(\begin{array}{l}
u_{1}^{n} \\
u_{2}^{n}
\end{array}\right)(t)-\left(\begin{array}{l}
R_{1} \\
R_{2}
\end{array}\right)(t)\right\|_{H^{1} \times H^{1}} \leqslant e^{-\sqrt{\omega_{*}} v_{*} t} .
$$

We prove that $t_{0}=T_{0}$ by contradiction. Assume that $t_{0}>T_{0}$. By Proposition [5] for all $t \in\left[t_{0}, T^{n}\right]$ we have

$$
\left\|\left(\begin{array}{l}
u_{1}^{n} \\
u_{2}^{n}
\end{array}\right)(t)-\left(\begin{array}{l}
R_{1} \\
R_{2}
\end{array}\right)(t)\right\|_{H^{1} \times H^{1}} \leqslant \frac{1}{2} e^{-\sqrt{\omega_{*}} v_{*} t} .
$$

Therefore by continuity there exists $t_{00}<t_{0}$ such that on $\left[t_{00}, T^{n}\right]$ estimate (9) is satisfied. This however contradicts the minimality of $t_{0}$. Hence $t_{0}=T_{0}$ and this concludes the proof.

For the rest of Section $3, T_{0}>0$ and $n_{0} \in \mathbb{N}$ will be large enough fixed numbers, and we assume the existence of $t_{0} \geqslant T_{0}$ such that for all $t \in\left[t_{0}, T^{n}\right]$ the bootstrap assumption (7) is verified, i.e. we have

$$
\left\|\left(\begin{array}{l}
u_{1}^{n} \\
u_{2}^{n}
\end{array}\right)(t)-\left(\begin{array}{l}
R_{1} \\
R_{2}
\end{array}\right)(t)\right\|_{H^{1} \times H^{1}} \leqslant e^{-\sqrt{\omega_{*}} v_{*} t} .
$$

Our final goal is now to prove that in fact (8) holds for all $t \in\left[t_{0}, T^{n}\right]$.

3.2. Modulation. Let us start with a decomposition lemma for our sequence of approximated multi-speed solitary waves.

Lemma 6 (Modulation). There exist $C>0$ and $\mathcal{C}^{1}$ functions

$$
\tilde{\omega}_{j}:\left[t_{0}, T^{n}\right] \rightarrow(0,+\infty), \quad \tilde{x}_{j}:\left[t_{0}, T^{n}\right] \rightarrow \mathbb{R}, \quad \tilde{\gamma}_{j}:\left[t_{0}, T^{n}\right] \rightarrow \mathbb{R}, \quad j=1,2,
$$

such that if for $j=1,2$ we denote by $\tilde{R}_{j}$ the modulated wave

$$
\tilde{R}_{j}(t, x)=e^{i\left(\frac{1}{2} v_{j} \cdot x+\tilde{\gamma}_{j}(t)\right)} \frac{1}{\sqrt{\mu_{j}}} Q_{\tilde{\omega}_{j}(t)}\left(x-\tilde{x}_{j}(t)\right),
$$

then for all $t \in\left[t_{0}, T^{n}\right]$ the functions defined by

$$
\left(\begin{array}{l}
\varepsilon_{1} \\
\varepsilon_{2}
\end{array}\right)(t)=\left(\begin{array}{l}
u_{1}^{n} \\
u_{2}^{n}
\end{array}\right)(t)-\left(\begin{array}{l}
\tilde{R}_{1} \\
\tilde{R}_{2}
\end{array}\right)(t)
$$

satisfy for $j=1,2$ and for all $t \in\left[t_{0}, T^{n}\right]$ the orthogonality conditions

$$
\left(\varepsilon_{j}(t), \tilde{R}_{j}(t)\right)_{2}=\left(\varepsilon_{j}(t), i \tilde{R}_{j}(t)\right)_{2}=\left(\varepsilon_{j}(t), \partial_{x} \tilde{R}_{j}(t)\right)_{2}=0 .
$$

Moreover, for all $t \in\left[t_{0}, T^{n}\right]$, we have

$$
\begin{aligned}
\sum_{j=1}^{2}\left(\left|\partial_{t} \tilde{\omega}_{j}(t)\right|^{2}+\left|\partial_{t} \tilde{x}_{j}(t)-v_{j}\right|^{2}+\right. & \left.\left|\partial_{t} \tilde{\gamma}_{j}(t)+\frac{v_{j}^{2}}{4}-\tilde{\omega}_{j}(t)\right|^{2}\right) \\
& \leqslant C\left\|\left(\begin{array}{l}
\varepsilon_{1} \\
\varepsilon_{2}
\end{array}\right)(t)\right\|_{H^{1} \times H^{1}}^{2}+C e^{-3 \sqrt{\omega_{*}} v_{*} t} .
\end{aligned}
$$


Remark 7. It is to be noticed that estimate (13) clearly implies that, for $T_{0}$ large enough, for $j=1,2$, and for all $t \in\left[t_{0}, T^{n}\right]$, we have

$$
\tilde{x}_{j}(t) \geqslant \frac{v_{*}}{2 \sqrt{2}} t>2 L \text { and } \tilde{\omega}_{j}(t) \geqslant 1152 \omega_{*} .
$$

Moreover, a better estimate can be obtained for $\tilde{\omega}_{j}$ and will be stated later on in Lemma 12 .

This type of modulation result is classical in the literature dealing with solitary waves of nonlinear dispersive equations (see e.g. the fundamental paper of Weinstein 31 for an early version or 24 for a recent approach). Its proof consists essentially in the application of the implicit function theorem combined with the use of the evolution equation to find equation (13) for the evolution of the modulation parameters. We refer to the appendix for the details of the proof.

3.3. Energy estimates and coercivity. In this subsection, we analyze the different quantities that are conserved or almost-conserved in our coupled-vectorial problem. Remember that in the case of the scalar equation (5) the energy, mass and momentum, defined as follows, are conserved along the flow of (5):

$$
E\left(u, \mu_{j}\right):=\frac{1}{2}\left\|\partial_{x} u\right\|_{L^{2}}^{2}-\frac{\mu_{j}}{4}\|u\|_{L^{4}}^{4}, \quad M(u):=\frac{1}{2}\|u\|_{L^{2}}^{2}, \quad P(u):=\frac{1}{2} \mathcal{I}_{m} \int_{\mathbb{R}} u \overline{\partial_{x} u} d x .
$$

The solution $Q_{\omega}$ we chose of equation (2) is known to be the unique positive radial ground state of the action $S:=E(\cdot, 1)+\omega M$. Consequently, each soliton $R_{j}$ defined by (4) is a critical point of the scalar functional $S_{j}$ defined by

$$
S_{j}:=E\left(\cdot, \mu_{j}\right)+\left(\omega_{j}+\frac{v_{j}^{2}}{4}\right) M+v_{j} P .
$$

Coercivity properties of linearizations of $S_{j}$-like functionals are the key tool of the analysis of multi-solitons interaction (see for example [17, 23]).

In the vectorial case we are interested in here, the coupled system (NLS) admits its own conservation laws. In particular, the mass of each component is preserved, as in the scalar case. However, the coupling does not preserve conservation of the scalar energy and momentum for each component and we only have conservation of the total energy (made of individual energies plus a coupling term) and total momentum (sum of the scalar momenta). More precisely, total energy, total momentum, and scalar masses of the whole system (defined as follows) are conserved quantities for the flow of system (NLS):

$$
\begin{gathered}
\mathcal{E}\left(\begin{array}{l}
u_{1} \\
u_{2}
\end{array}\right):=E\left(u_{1}, \mu_{1}\right)+E\left(u_{2}, \mu_{2}\right)-\frac{\beta}{2} \int_{\mathbb{R}}\left|u_{1}\right|^{2}\left|u_{2}\right|^{2} d x, \\
\mathcal{P}\left(\begin{array}{l}
u_{1} \\
u_{2}
\end{array}\right):=P\left(u_{1}\right)+P\left(u_{2}\right), \quad M_{j}\left(\begin{array}{l}
u_{1} \\
u_{2}
\end{array}\right):=M\left(u_{j}\right), \quad j=1,2 .
\end{gathered}
$$

In order to use the conservation of the momentum as in the scalar case, we here need to localize the momentum of each soliton, as was done in [12, 13] for the scalar mass and momentum. Note that this was not needed for the analysis in [17]. Let us define the cut-off functions

$$
\chi_{L}^{1}(x)=\chi\left(\frac{x}{L}\right), \quad \chi_{L}^{2}=1-\chi_{L}^{1},
$$


where $L>0$ is arbitrary but fixed and $\chi$ is a $\mathcal{C}^{3}$ function such that

$$
0 \leqslant \chi \leqslant 1 \text { on } \mathbb{R}, \quad \chi(x)=0 \text { for } x \leqslant-1, \quad \chi(x)=1 \text { for } x>1, \quad \chi^{\prime} \geqslant 0 \text { on } \mathbb{R},
$$

and satisfies, for some positive constant $C$ and for all $x \in \mathbb{R}$ the estimates

$$
\left(\chi^{\prime}(x)\right)^{2} \leqslant C \chi(x), \quad\left(\chi^{\prime \prime}(x)\right)^{2} \leqslant C \chi^{\prime}(x) .
$$

Localized momenta $\mathcal{P}_{\text {loc }}^{j}$ are defined by:

$$
\mathcal{P}_{\text {loc }}^{j}\left(\begin{array}{l}
u_{1} \\
u_{2}
\end{array}\right)=\frac{1}{2} \mathcal{I} m \int_{\mathbb{R}}\left(u_{1} \overline{\partial_{x} u_{1}}+u_{2} \overline{\partial_{x} u_{2}}\right) \chi_{L}^{j} d x, \quad j=1,2 .
$$

Remark that $\mathcal{P}=\mathcal{P}_{\text {loc }}^{1}+\mathcal{P}_{\text {loc }}^{2}$. Note that since we are assuming (6) the momenta above defined are localized around each composing solitary wave of the profile. The advantage of having made assumption (6) is that the cut-off does not depend on time. This will simplify our next calculations.

In the sequel, we are interested in the following global action:

$$
\begin{array}{r}
\mathcal{S}\left(\begin{array}{l}
u_{1} \\
u_{2}
\end{array}\right)=\mathcal{E}\left(\begin{array}{l}
u_{1} \\
u_{2}
\end{array}\right)+\sum_{j=1,2}\left(\tilde{\omega}_{j}(t)+\frac{v_{j}^{2}}{4}\right) M_{j}\left(\begin{array}{l}
u_{1} \\
u_{2}
\end{array}\right)+\sum_{j=1,2} v_{j} \mathcal{P}_{\text {loc }}^{j}\left(\begin{array}{l}
u_{1} \\
u_{2}
\end{array}\right) \\
+C_{1}\left(\beta, v_{1}\right) M_{2}\left(\begin{array}{l}
u_{1} \\
u_{2}
\end{array}\right)+C_{2}\left(\beta, v_{2}\right) M_{1}\left(\begin{array}{l}
u_{1} \\
u_{2}
\end{array}\right),
\end{array}
$$

where $C_{j}\left(\beta, v_{j}\right)$ are positive constants depending only on $\beta$ and $v_{j}$ and whose exact values will be decided later on. Note that the action implicitly depends on $t$ via $\tilde{\omega}_{j}$. It is to be noted that, in this work, we have the freedom to add these two coupled-mass terms that do not appear in the usual definition of $\mathcal{S}$-like functionals. This is a key point in our analysis.

Let us now state in the following lemma several estimates related to the localization of $\tilde{R}_{1}$ and $\tilde{R}_{2}$ and which will be of great use in the sequel.

Lemma 8. For $j=1,2$, if $T_{0}$ is large enough, then for all $t \in\left[t_{0}, T^{n}\right]$ and for all $x \in \mathbb{R}$ we have

$$
\begin{aligned}
\left(\left|\tilde{R}_{j}(t, x)\right|+\left|\partial_{x} \tilde{R}_{j}(t, x)\right|\right) \chi_{L}^{3-j}(x) & \leqslant C\left(1+\left|v_{j}\right|\right) e^{-3 \sqrt{\omega_{*}} v_{*} t} e^{-\sqrt{\omega_{*}}|x|}, \\
\prod_{k=1,2}\left(\left|\tilde{R}_{k}(t, x)\right|+\left|\partial_{x} \tilde{R}_{k}(t, x)\right|\right) & \leqslant C\left(1+\left|v_{1}\right|+\left|v_{2}\right|\right)^{2} e^{-3 \sqrt{\omega_{*}} v_{*} t} e^{-\sqrt{\omega_{*}}|x|} .
\end{aligned}
$$

Lemma 8 follows from the support properties of the cut-off function and the exponential localization of the solitons profiles. Indeed, recall that in fact, the profile $Q=Q_{1}$ is explicitly known

$$
Q(x)=2 \operatorname{sech}(x),
$$

and it follows that $Q$ and its derivatives are exponentially decaying, i.e. for any $\eta<1$ we have

$$
\left|Q_{x x}\right|+\left|Q_{x}\right|+|Q| \leqslant C_{\eta} e^{-\eta|x|} .
$$

Proof of Lemma 8. We prove only (16), the proof of (17) following from similar (simpler) arguments. For simplicity in notation, assume $j=1$, the case $j=2$ being perfectly symmetric. Due to the exponential decay of the soliton profiles, we have

$$
\left|\tilde{R}_{1}(t, x)\right| \leqslant C e^{-\frac{3}{4} \sqrt{\omega_{1}}\left|x-\tilde{x}_{1}\right|}
$$


The cut-off function $\chi_{L}^{2}$ is supported on $(-\infty, L]$, and since for $T_{0}$ large enough $\tilde{x}_{1}>2 L$, for $x \in(-\infty, L]$ we have (we recall here Remark 7 )

$$
\left|x-\tilde{x}_{1}\right| \geqslant|x|, \quad \text { and } \quad\left|x-\tilde{x}_{1}\right| \geqslant \frac{1}{2}\left|\tilde{x}_{1}\right| .
$$

As a consequence, we have

$$
\left|\tilde{R}_{1}(t, x)\right| \chi_{L}^{2}(x) \leqslant C e^{-\frac{1}{4} \sqrt{\omega_{1}}\left|\tilde{x}_{1}\right|} e^{-\frac{1}{4} \sqrt{\omega_{1}}|x|} .
$$

In addition, as noticed in Remark 7 , we have

which implies

$$
\tilde{x}_{1} \geqslant \frac{v_{*}}{2 \sqrt{2}} t, \quad \text { and } \quad \tilde{\omega}_{1} \geqslant 1152 \omega_{*}
$$

$$
\left|\tilde{R}_{1}(t, x)\right| \chi_{L}^{2}(x) \leqslant C e^{-3 \sqrt{\omega_{*}} v_{*} t} e^{-\sqrt{\omega_{*}}|x|} .
$$

The derivative $\partial_{x} \tilde{R}_{1}$ is treated in the same way, with the only difference that $\left|v_{1}\right|$ now appears in the estimate, due to the term $e^{i \frac{1}{2} v_{1} \cdot x}$ in the definition (11) of $\tilde{R}_{1}$. This finishes the proof.

Lemma 9 (Expansion of the global action $\mathcal{S}$ ). For all $t \in\left[t_{0}, T^{n}\right]$ we have

$$
\begin{gathered}
\mathcal{S}\left(\begin{array}{l}
u_{1} \\
u_{2}
\end{array}\right)=\mathcal{S}\left(\begin{array}{l}
\tilde{R}_{1} \\
\tilde{R}_{2}
\end{array}\right)+\mathcal{H}\left(\begin{array}{l}
\varepsilon_{1} \\
\varepsilon_{2}
\end{array}\right)+\mathcal{O}\left(e^{-3 \sqrt{\omega_{*}} v_{*} t}\right), \\
\mathcal{H}\left(\begin{array}{l}
\varepsilon_{1} \\
\varepsilon_{2}
\end{array}\right)=\mathcal{H}_{\text {free }}\left(\begin{array}{l}
\varepsilon_{1} \\
\varepsilon_{2}
\end{array}\right)+\mathcal{H}_{\text {coupled }}\left(\begin{array}{l}
\varepsilon_{1} \\
\varepsilon_{2}
\end{array}\right),
\end{gathered}
$$

with

$$
\begin{aligned}
\mathcal{H}_{\text {free }}\left(\begin{array}{c}
\varepsilon_{1} \\
\varepsilon_{2}
\end{array}\right)=\sum_{j=1,2}\left(\frac{1}{2}\left\|\partial_{x} \varepsilon_{j}\right\|_{L^{2}}^{2}\right. & +\frac{1}{2}\left(\tilde{\omega}_{j}+\frac{v_{j}^{2}}{4}\right)\left\|\varepsilon_{j}\right\|_{L^{2}}^{2}-\mu_{j} \int_{\mathbb{R}}\left|\varepsilon_{j}\right|^{2}\left|\tilde{R}_{j}\right|^{2} d x \\
& \left.-\frac{\mu_{j}}{2} \mathcal{R} e \int_{\mathbb{R}} \varepsilon_{j}^{2} \overline{\tilde{R}_{j}^{2}} d x+\frac{1}{2} v_{j} \mathcal{I}_{m} \int_{\mathbb{R}} \varepsilon_{j} \overline{\partial_{x} \varepsilon_{j}} \chi_{L}^{j} d x\right)
\end{aligned}
$$

and

$$
\begin{gathered}
\mathcal{H}_{\text {coupled }}\left(\begin{array}{l}
\varepsilon_{1} \\
\varepsilon_{2}
\end{array}\right)=C_{1}\left(\beta, v_{1}\right)\left\|\varepsilon_{2}\right\|_{L^{2}}^{2}+C_{2}\left(\beta, v_{2}\right)\left\|\varepsilon_{1}\right\|_{L^{2}}^{2} \\
-\frac{\beta}{2} \int_{\mathbb{R}}\left(\left|\varepsilon_{1}\right|^{2}\left|\tilde{R}_{2}\right|^{2}+\left|\varepsilon_{2}\right|^{2}\left|\tilde{R}_{1}\right|^{2}\right) d x \\
\quad+\frac{1}{2} v_{1} \mathcal{I}_{m} \int_{\mathbb{R}} \varepsilon_{2} \overline{\partial_{x} \varepsilon_{2}} \chi_{L}^{1} d x+\frac{1}{2} v_{2} \mathcal{I} m \int_{\mathbb{R}} \varepsilon_{1} \overline{\partial_{x} \varepsilon_{1}} \chi_{L}^{2} d x .
\end{gathered}
$$

Proof of Lemma 9. First note that, according to the definition (15) of $\mathcal{S}$,

$$
\begin{aligned}
\mathcal{S}\left(\begin{array}{l}
u_{1} \\
u_{2}
\end{array}\right)=\sum_{j=1,2} \tilde{S}_{j}\left(u_{j}\right)+v_{j} \cdot\left(\mathcal{P}_{\text {loc }}^{j}\left(\begin{array}{l}
u_{1} \\
u_{2}
\end{array}\right)-P\left(u_{i}\right)\right) \\
\\
\quad+C_{1}\left(\beta, v_{1}\right) M\left(u_{1}\right)+C_{2}\left(\beta, v_{2}\right) M\left(u_{2}\right)-\frac{\beta}{2} \int_{\mathbb{R}}\left|u_{1}\right|^{2}\left|u_{2}\right|^{2} d x,
\end{aligned}
$$

where $\tilde{S}_{j}$ denote the same functional as $S_{j}$ (see the definition (14)) with $\tilde{\omega}_{j}$ instead of $\omega_{j}$. For $j=1,2$, let us expand $u_{j}(t)=\tilde{R}_{j}(t)+\varepsilon_{j}(t)$ in the expression of $\tilde{S}_{j}$. A simple computation leads to:

$$
\tilde{S}_{j}\left(\tilde{R}_{j}+\varepsilon_{j}\right)=\tilde{S}_{j}\left(\tilde{R}_{j}\right)+\tilde{S}_{j}^{\prime}\left(\tilde{R}_{j}\right) \varepsilon_{j}+\left\langle\tilde{S}_{j}^{\prime \prime}\left(\tilde{R}_{j}\right) \varepsilon_{j}, \varepsilon_{j}\right\rangle+\mathcal{O}\left(\left\|\varepsilon_{j}\right\|_{H^{1}}^{3}\right)
$$


Now, as $\tilde{R}_{j}$ is a critical point of the scalar functional $\tilde{S}_{j}$, we have

$$
\tilde{S}_{j}^{\prime}\left(\tilde{R}_{j}\right)=0
$$

and thus

$$
\tilde{S}_{j}\left(\tilde{R}_{j}+\varepsilon_{j}\right)=\tilde{S}_{j}\left(\tilde{R}_{j}\right)+\left\langle\tilde{S}_{j}^{\prime \prime}\left(\tilde{R}_{j}\right) \varepsilon_{j}, \varepsilon_{j}\right\rangle+\mathcal{O}\left(\left\|\varepsilon_{j}\right\|_{H^{1}}^{3}\right)
$$

where

$$
\begin{aligned}
\left\langle\tilde{S}_{j}^{\prime \prime}\left(\tilde{R}_{j}\right) \varepsilon_{j}, \varepsilon_{j}\right\rangle=\frac{1}{2}\left\|\partial_{x} \varepsilon_{j}\right\|_{L^{2}}^{2}+\frac{1}{2} & \left(\tilde{\omega}_{j}+\frac{v_{j}^{2}}{4}\right)\left\|\varepsilon_{j}\right\|_{L^{2}}^{2}+\frac{1}{2} v_{j} \mathcal{I}_{m} \int_{\mathbb{R}} \varepsilon_{j} \overline{\partial_{x} \varepsilon_{j}} d x \\
& -\frac{\mu_{j}}{2} \mathcal{R} e \int_{\mathbb{R}} \varepsilon_{j}^{2} \overline{\tilde{R}_{j}^{2}} d x-\mu_{j} \int_{\mathbb{R}}\left|\varepsilon_{j}\right|^{2}\left|\tilde{R}_{j}\right|^{2} d x
\end{aligned}
$$

Let us now develop the remaining terms in (19). As far as the momentum part is concerned, we write the expansion for $j=1$ for simplicity:

$$
\begin{aligned}
& \mathcal{P}_{\text {loc }}^{1}\left(\begin{array}{l}
\tilde{R}_{1}+\varepsilon_{1} \\
\tilde{R}_{2}+\varepsilon_{2}
\end{array}\right)-P\left(\tilde{R}_{1}+\varepsilon_{1}\right) \\
& =-\mathcal{I}_{m} \int_{\mathbb{R}} \tilde{R}_{1} \overline{\partial_{x} \tilde{R}_{1}} \chi_{L}^{2} d x+\mathcal{I}_{m} \int_{\mathbb{R}} \tilde{R}_{2} \overline{\partial_{x} \tilde{R}_{2}} \chi_{L}^{1} d x \\
& \quad-\mathcal{I}_{m} \int_{\mathbb{R}}\left(\tilde{R}_{1} \overline{\partial_{x} \varepsilon_{1}}+\varepsilon_{1} \overline{\partial_{x} \tilde{R}_{1}}\right) \chi_{L}^{2} d x+\mathcal{I}_{m} \int_{\mathbb{R}}\left(\tilde{R}_{2} \overline{\partial_{x} \varepsilon_{2}}+\varepsilon_{2} \overline{\partial_{x} \tilde{R}_{2}}\right) \chi_{L}^{1} d x \\
& \quad-\mathcal{I}_{m} \int_{\mathbb{R}} \varepsilon_{1} \overline{\partial_{x} \varepsilon_{1}} \chi_{L}^{2} d x+\mathcal{I}_{m} \int_{\mathbb{R}} \varepsilon_{2} \overline{\partial_{x} \varepsilon_{2}} \chi_{L}^{1} d x .
\end{aligned}
$$

Concerning the $\beta$ coupling part in (19), we get:

$$
\begin{aligned}
& \int_{\mathbb{R}}\left|\tilde{R}_{1}+\varepsilon_{1}\right|^{2}\left|\tilde{R}_{2}+\varepsilon_{2}\right|^{2} d x \\
& =\int_{\mathbb{R}}\left|\tilde{R}_{1}\right|^{2}\left|\tilde{R}_{2}\right|^{2} d x+2 \mathcal{R} e \int_{\mathbb{R}}\left(\left|\tilde{R}_{1}\right|^{2} \overline{\tilde{R}_{2}} \varepsilon_{2}+\left|\tilde{R}_{2}\right|^{2} \overline{\tilde{R}_{1}} \varepsilon_{1}\right) d x \\
& \quad+\int_{\mathbb{R}}\left(\left|\varepsilon_{1}\right|^{2}\left|\tilde{R}_{2}\right|^{2}+\left|\varepsilon_{2}\right|^{2}\left|\tilde{R}_{1}\right|^{2}+4 \operatorname{Re}\left(\varepsilon_{1} \tilde{R}_{1}\right) \operatorname{Re}\left(\varepsilon_{2} \tilde{R}_{2}\right)\right) d x \\
& \quad+2 \int_{\mathbb{R}}\left(\left|\varepsilon_{2}\right|^{2} \operatorname{Re}\left(\varepsilon_{1} \overline{\tilde{R}_{1}}\right)+\left|\varepsilon_{1}\right|^{2} \operatorname{Re}\left(\varepsilon_{2} \overline{\tilde{R}_{2}}\right)\right) d x+\int_{\mathbb{R}}\left|\varepsilon_{1}\right|^{2}\left|\varepsilon_{2}\right|^{2} d x .
\end{aligned}
$$

Finally, the extra-masses terms in (19) expand into

$$
M\left(\tilde{R}_{j}+\varepsilon_{j}\right)=\frac{1}{2}\left\|\tilde{R}_{j}+\varepsilon_{j}\right\|_{L^{2}}^{2}=M\left(\tilde{R}_{j}\right)+\left(\tilde{R}_{j}, \varepsilon_{j}\right)_{2}+M\left(\varepsilon_{j}\right)=M\left(\tilde{R}_{j}\right)+M\left(\varepsilon_{j}\right),
$$

where we have used the orthogonality conditions (12) to obtain the last equality. In (22) and (23), all terms containing a product of solitons or cut-off functions with different indices/exponents are of order $\mathcal{O}\left(e^{-3 \sqrt{\omega_{*}} v_{*} t}\right)$ by Lemma 8 . The terms containing a degree 3 or higher term in $\left(\varepsilon_{1}, \varepsilon_{2}\right)$ are also of order $\mathcal{O}\left(e^{-3 \sqrt{\omega_{*}} v_{*} t}\right)$ by the bootstrap assumption (10). Therefore, gathering (19)-(20)-(21)-(22)-(23)-(24) together gives:

$$
\mathcal{S}\left(\begin{array}{l}
\tilde{R}_{1}+\varepsilon_{1} \\
\tilde{R}_{2}+\varepsilon_{2}
\end{array}\right)=\mathcal{S}\left(\begin{array}{l}
\tilde{R}_{1} \\
\tilde{R}_{2}
\end{array}\right)+\mathcal{H}_{\text {free }}\left(\begin{array}{l}
\varepsilon_{1} \\
\varepsilon_{2}
\end{array}\right)+\mathcal{H}_{\text {coupled }}\left(\begin{array}{l}
\varepsilon_{1} \\
\varepsilon_{2}
\end{array}\right)+\mathcal{O}\left(e^{-3 \sqrt{\omega_{*}} v_{*} t}\right) .
$$

This concludes the proof. 
Lemma 10 (Coercivity of $\mathcal{H}$ ). There exists $\lambda>0$ such that, for any $t_{0} \in\left[T_{0}, T^{n}\right]$ and for all $t \in\left[t_{0}, T^{n}\right]$ :

$$
\begin{gathered}
\mathcal{H}_{\text {free }}\left(\begin{array}{c}
\varepsilon_{1} \\
\varepsilon_{2}
\end{array}\right) \geqslant 2 \lambda\left(\left\|\varepsilon_{1}\right\|_{H^{1}}^{2}+\left\|\varepsilon_{2}\right\|_{H^{1}}^{2}\right), \\
\mathcal{H}_{\text {coupled }}\left(\begin{array}{l}
\varepsilon_{1} \\
\varepsilon_{2}
\end{array}\right) \geqslant \lambda\left(\left\|\varepsilon_{1}\right\|_{L^{2}}^{2}+\left\|\varepsilon_{2}\right\|_{L^{2}}^{2}\right)-\lambda\left(\left\|\partial_{x} \varepsilon_{1}\right\|_{L^{2}}^{2}+\left\|\partial_{x} \varepsilon_{2}\right\|_{L^{2}}^{2}\right),
\end{gathered}
$$

and thus:

$$
\mathcal{H}\left(\begin{array}{l}
\varepsilon_{1} \\
\varepsilon_{2}
\end{array}\right) \geqslant \lambda\left\|\left(\begin{array}{l}
\varepsilon_{1} \\
\varepsilon_{2}
\end{array}\right)\right\|_{H^{1} \times H^{1}}^{2} .
$$

Proof. The proof of (25) is classical (see for example [17, 23]) and we omit it. It remains to prove (26). It is readily seen that for $C_{j}\left(\beta, v_{j}\right)$ large enough, we have

$$
\begin{aligned}
C_{1}\left(\beta, v_{1}\right)\left\|\varepsilon_{2}\right\|_{L^{2}}^{2}+C_{2}\left(\beta, v_{2}\right)\left\|\varepsilon_{1}\right\|_{L^{2}}^{2} & -\frac{\beta}{2} \int_{\mathbb{R}}\left(\left|\varepsilon_{1}\right|^{2}\left|\tilde{R}_{2}\right|^{2}+\left|\varepsilon_{2}\right|^{2}\left|\tilde{R}_{1}\right|^{2}\right) d x \\
& \geqslant \frac{1}{2}\left(C_{1}\left(\beta, v_{1}\right)\left\|\varepsilon_{2}\right\|_{L^{2}}^{2}+C_{2}\left(\beta, v_{2}\right)\left\|\varepsilon_{1}\right\|_{L^{2}}^{2}\right) .
\end{aligned}
$$

As far as the momentum parts of $\mathcal{H}_{\text {coupled }}$ are concerned, for example for $j=1$ :

$$
\begin{aligned}
\frac{v_{1}}{2} \mathcal{I}_{m} \int_{R} \varepsilon_{1} \overline{\partial_{x} \varepsilon_{1}} \chi_{L}^{2} d x & \geqslant-\frac{v_{1}}{2} \int_{\mathbb{R}}\left|\varepsilon_{1} \partial_{x} \varepsilon_{1} \chi_{L}^{2}\right| d x \\
& \geqslant-\frac{v_{1}}{2}\left\|\varepsilon_{1}\right\|_{2}\left\|\partial_{x} \varepsilon_{1}\right\|_{2} \\
& \geqslant-\frac{v_{1}^{2}}{4 \lambda}\left\|\varepsilon_{1}\right\|_{L^{2}}^{2}-\lambda\left\|\partial_{x} \varepsilon_{1}\right\|_{L^{2}}^{2} .
\end{aligned}
$$

For $C\left(\beta, v_{j}\right), j=1,2$ large enough, i.e such that

$$
\sum_{j=1,2}\left(\frac{1}{2} C\left(\beta, v_{j}\right)-\frac{v_{j}^{2}}{4 \lambda}\right)>\lambda,
$$

we thus have:

$$
\mathcal{H}_{\text {coupled }}\left(\begin{array}{l}
\varepsilon_{1} \\
\varepsilon_{2}
\end{array}\right) \geqslant \lambda\left(\left\|\varepsilon_{1}\right\|_{L^{2}}^{2}+\left\|\varepsilon_{2}\right\|_{L^{2}}^{2}\right)-\lambda\left(\left\|\partial_{x} \varepsilon_{1}\right\|_{L^{2}}^{2}+\left\|\partial_{x} \varepsilon_{2}\right\|_{L^{2}}^{2}\right) .
$$

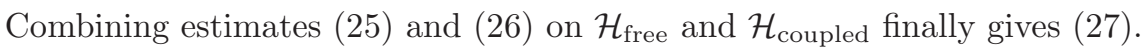

3.4. Almost-conservation of the localized momentum. In this section, we investigate the conservation of the localized momentum and, inspired by [23], we state the following lemma:

Lemma 11. There exists $C>0$ (independent of $L$ ) such that if $L$ and $T_{0}$ are large enough then for all $t \in\left[t_{0}, T^{n}\right]$ and for $j=1,2$ we have

$$
\left|\mathcal{P}_{\text {loc }}^{j}\left(\begin{array}{l}
u_{1} \\
u_{2}
\end{array}\right)(t)-\mathcal{P}_{\text {loc }}^{j}\left(\begin{array}{l}
u_{1} \\
u_{2}
\end{array}\right)\left(T^{n}\right)\right| \leqslant \frac{C}{L} e^{-2 \sqrt{\omega_{*}} v_{*} t} .
$$

Proof of Lemma 11. Let us prove the lemma in the case $j=1$, the case $j=2$ being perfectly similar. We first compute the time derivative of $\mathcal{P}_{\text {loc }}^{1}$ :

$$
\frac{d}{d t} \mathcal{P}_{\text {loc }}^{1}(t)=\frac{1}{2} \mathcal{I}_{m} \int_{\mathbb{R}} \partial_{t} u_{1} \partial_{x} \overline{u_{1}} \chi\left(\frac{x}{L}\right) d x+\frac{1}{2} \mathcal{I}_{m} \int_{\mathbb{R}} u_{1} \partial_{x, t} \overline{u_{1}} \chi\left(\frac{x}{L}\right) d x .
$$


Let us call term $A$ and $B$ respectively the first and the second term of the right hand side in equality (29):

$$
A=\frac{1}{2} \mathcal{I}_{m} \int_{\mathbb{R}} \partial_{t} u_{1} \partial_{x} \overline{u_{1}} \chi\left(\frac{x}{L}\right) d x, \quad B=\frac{1}{2} \mathcal{I}_{m} \int_{\mathbb{R}} u_{1} \partial_{x} \partial_{t} \overline{u_{1}} \chi\left(\frac{x}{L}\right) d x .
$$

Using the fact that $u(t)=\left(\begin{array}{l}u_{1}(t) \\ u_{2}(t)\end{array}\right)$ is a solution to (1NLS) , we readily get (formally, but this will be justified when integrating):

$$
\begin{aligned}
& \partial_{t} u_{1} \partial_{x} \overline{u_{1}}=i \partial_{x x} u_{1} \partial_{x} \overline{u_{1}}+i \mu_{1}\left|u_{1}\right|^{2} u_{1} \partial_{x} \overline{u_{1}}+i \beta\left|u_{2}\right|^{2} u_{1} \partial_{x} \overline{u_{1}} \\
& \partial_{x} \partial_{t} \overline{u_{1}}=-i \partial_{x x x} \overline{u_{1}}-i \mu_{1} \partial_{x}\left(\left|u_{1}\right|^{2} \overline{u_{1}}\right)-i \beta \partial_{x}\left(\left|u_{2}\right|^{2} \overline{u_{1}}\right) .
\end{aligned}
$$

About term A: Equation (30) provides us with the following decomposition of term $A$ :

$$
\begin{aligned}
A=\frac{1}{2} \mathcal{R} e \int_{\mathbb{R}} \partial_{x x} u_{1} \partial_{x} \overline{u_{1}} \chi\left(\frac{x}{L}\right) d x+\frac{1}{2} \mu_{1} \mathcal{R} e \int_{\mathbb{R}}\left|u_{1}\right|^{2} u_{1} \partial_{x} \overline{u_{1}} \chi\left(\frac{x}{L}\right) d x \\
+\frac{1}{2} \beta \mathcal{R} e \int_{\mathbb{R}}\left|u_{2}\right|^{2} u_{1} \partial_{x} \overline{u_{1}} \chi\left(\frac{x}{L}\right) d x .
\end{aligned}
$$

Integrating by part in each term of $A$ finally gives:

$$
\begin{aligned}
A=-\frac{1}{4 L} \int_{\mathbb{R}}\left|\partial_{x} u_{1}\right|^{2} \chi^{\prime}\left(\frac{x}{L}\right) d x-\frac{1}{8 L} \mu_{1} \int_{\mathbb{R}}\left|u_{1}\right|^{4} \chi^{\prime}\left(\frac{x}{L}\right) d x \\
+\frac{1}{2} \beta \mathcal{R e} \int_{\mathbb{R}}\left|u_{2}\right|^{2} u_{1} \partial_{x} \overline{u_{1}} \chi\left(\frac{x}{L}\right) d x .
\end{aligned}
$$

About term B: Equation (31) provides us with the following decomposition of term $B$ :

$$
\begin{aligned}
B=-\frac{1}{2} \mathcal{R} e \int_{\mathbb{R}} u_{1} \partial_{x x x} \overline{u_{1}} \chi\left(\frac{x}{L}\right) d x-\frac{1}{2} \mu_{1} \mathcal{R} \boldsymbol{e} \int_{\mathbb{R}} u_{1} \partial_{x}\left(\left|u_{1}\right|^{2} \overline{u_{1}}\right) \chi\left(\frac{x}{L}\right) d x \\
\quad-\frac{1}{2} \beta \mathcal{R} \boldsymbol{e} \int_{\mathbb{R}} u_{1} \partial_{x}\left(\left|u_{2}\right|^{2} \overline{u_{1}}\right) \chi\left(\frac{x}{L}\right) d x .
\end{aligned}
$$

As for the $A$ term, integrating by parts (several times if necessary) in each term finally leads to:

$$
\begin{aligned}
B=-\frac{3}{4 L} \int_{\mathbb{R}}\left|\partial_{x} u_{1}\right|^{2} \chi^{\prime}\left(\frac{x}{L}\right) d x & +\frac{1}{4 L^{3}} \int_{\mathbb{R}}\left|u_{1}\right|^{2} \chi^{\prime \prime \prime}\left(\frac{x}{L}\right) d x \\
& +\frac{3}{8 L} \mu_{1} \int_{\mathbb{R}}\left|u_{1}\right|^{4} \chi^{\prime}\left(\frac{x}{L}\right) d x \\
& +\frac{1}{2} \beta \mathcal{R} e \int_{\mathbb{R}}\left|u_{2}\right|^{2} u_{1} \partial_{x} \overline{u_{1}} \chi\left(\frac{x}{L}\right) d x+\frac{1}{2 L} \beta \mathcal{R} e \int_{\mathbb{R}}\left|u_{2}\right|^{2}\left|u_{1}\right|^{2} \chi^{\prime}\left(\frac{x}{L}\right) d x .
\end{aligned}
$$

Combining (32) and (33), and using the fact that $\chi^{\prime}$ and $\chi^{\prime \prime \prime}$ are supported on $[-L, L]$ lead to the following estimate:

$\left|\frac{d}{d t} \mathcal{P}_{\text {loc }}^{1}(t)\right| \leqslant \frac{C}{L} \int_{-L}^{L}\left(\left|\partial_{x} u_{1}\right|^{2}+\left|u_{1}\right|^{2}+\left|u_{1}\right|^{4}\right) d x+C \int_{\mathbb{R}}\left|u_{2}\right|^{2}\left(\left|u_{1}\right|^{2}+\left|\partial_{x} u_{1}\right|^{2}\right) \chi_{L}^{1}(x) d x$. 
Note that for $x \in[-L, L], T_{0}$ large enough, and using (13) we have (see Lemma 8 for similar arguments)

$$
\left|\partial_{x} R_{j}(t, x)+R_{j}(t, x)\right| \leqslant C e^{-\sqrt{\tilde{\omega}_{j}}\left|x-\tilde{x}_{j}\right|} \leqslant C e^{-\frac{1}{2} \sqrt{\tilde{\omega}_{j} \mid}\left|\tilde{x}_{j}\right|} \leqslant C e^{-3 \sqrt{\omega_{*}} v_{*} t} .
$$

Note that $C$ here depends on $v_{j}$. Expanding now $u_{j}=\tilde{R}_{j}+\varepsilon_{j}$, in (34), using the above estimate and Lemma 8, we obtain

$$
\left|\frac{d}{d t} \mathcal{P}_{\mathrm{loc}}^{1}(t)\right| \leqslant \frac{C}{L}\left\|\left(\begin{array}{l}
\varepsilon_{1} \\
\varepsilon_{2}
\end{array}\right)\right\|_{H^{1} \times H^{1}}^{2}+\mathcal{O}\left(e^{-3 \sqrt{\omega_{*}} v_{*} t}\right) .
$$

The result follows integrating in time between $t$ and $T^{n}$ and using the bootstrap assumption (10).

3.5. Control of the modulation parameters. We now give an estimate of the variations of $\tilde{\omega}_{1}$ with respect to time. This estimate is better than (13) given by the modulation lemma.

Lemma 12 (Variations of $\left.\tilde{\omega}_{j}(t)\right)$. For $j=1,2$ and for all $t \in\left[t_{0}, T^{n}\right]$, we have

$$
\left|\tilde{\omega}_{j}(t)-\omega_{j}\right| \leqslant C\left\|\varepsilon_{j}(t)\right\|_{L^{2}}^{2} .
$$

Proof. This estimate is due to the choice of the modulation orthogonality condition $\left(\varepsilon_{j}(t), \tilde{R}_{j}(t)\right)_{2}=0$, and the conservation of the mass of each component:

$$
\begin{aligned}
0=\left\|u_{j}\right\|_{2}^{2}-\left\|R_{j}\right\|_{2}^{2}=\left\|\tilde{R}_{j}\right\|_{2}^{2}-\left\|R_{j}\right\|_{2}^{2}+\left\|\varepsilon_{j}\right\|_{2}^{2} & \\
& =\left(\tilde{\omega}_{j}-\omega_{j}\right) \frac{\partial}{\partial \omega}{ }_{\mid \omega=\omega_{j}}\left\|Q_{\omega}\right\|_{2}^{2}+\mathcal{O}\left(\left|\tilde{\omega}_{j}-\omega_{j}\right|^{2}\right)+\left\|\varepsilon_{j}\right\|_{2}^{2} .
\end{aligned}
$$

Since $\frac{\partial}{\partial \omega}\left|\omega=\omega_{j}\right| Q_{\omega} \|_{2}^{2}<0$, this concludes the proof.

3.6. Conclusion. With the elements of the previous subsections in hand, we can now conclude the proof of Proposition 5 .

End of the proof of Proposition [5. Recall that we have made the bootstrap assumption (10) and that our goal is to prove that for all $t \in\left[t_{0}, T^{n}\right]$ we have in fact the better estimate

$$
\left\|\left(\begin{array}{l}
u_{1}^{n} \\
u_{2}^{n}
\end{array}\right)-\left(\begin{array}{l}
R_{1} \\
R_{2}
\end{array}\right)\right\|_{H^{1} \times H^{1}} \leqslant \frac{1}{2} e^{-\sqrt{\omega_{*}} v_{*} t}
$$

Let us first expand

$$
\begin{array}{r}
\left\|\left(\begin{array}{l}
u_{1}^{n}(t) \\
u_{2}^{n}(t)
\end{array}\right)-\left(\begin{array}{l}
R_{1}(t) \\
R_{2}(t)
\end{array}\right)\right\|_{H^{1} \times H^{1}} \\
\leqslant\left\|\left(\begin{array}{l}
u_{1}^{n}(t) \\
u_{2}^{n}(t)
\end{array}\right)-\left(\begin{array}{c}
\tilde{R}_{1}(t) \\
\tilde{R}_{2}(t)
\end{array}\right)\right\|_{H^{1} \times H^{1}}+\left\|\left(\begin{array}{c}
\tilde{R}_{1}(t) \\
\tilde{R}_{2}^{n}(t)
\end{array}\right)-\left(\begin{array}{l}
R_{1}(t) \\
R_{2}(t)
\end{array}\right)\right\|_{H^{1} \times H^{1}} \\
\leqslant\left\|\left(\begin{array}{c}
\varepsilon_{1}(t) \\
\varepsilon_{2}(t)
\end{array}\right)\right\|_{H^{1} \times H^{1}}+C \sum_{j=1,2}\left|\tilde{\omega}_{j}-\omega_{j}\right|+\sum_{j=1,2} \mathcal{O}\left(\left|\tilde{\omega}_{j}-\omega_{j}\right|^{2}\right) .
\end{array}
$$

By Lemma 12, the part involving $\left|\tilde{\omega}_{j}-\omega_{j}\right|$ is controlled by the $\varepsilon$-part. Hence to finish the proof it is sufficient to control $\varepsilon$. Using successively the coercivity of $\mathcal{H}$ 
given in (27), the expansion (18) of the global action $\mathcal{S}$, the conservation of energy and mass, and the almost conservation of localized momenta (28), we obtain

$$
\begin{aligned}
\left\|\left(\begin{array}{l}
\varepsilon_{1}(t) \\
\varepsilon_{2}(t)
\end{array}\right)\right\|_{H^{1} \times H^{1}}^{2} & \leqslant C \mathcal{H}\left(\begin{array}{l}
\varepsilon_{1}(t) \\
\varepsilon_{2}(t)
\end{array}\right) \\
& \leqslant \mathcal{S}\left(\begin{array}{l}
u_{1}(t) \\
u_{2}(t)
\end{array}\right)-\mathcal{S}\left(\begin{array}{l}
\tilde{R}_{1}(t) \\
\tilde{R}_{2}(t)
\end{array}\right)+\mathcal{O}\left(e^{-3 \sqrt{\omega_{*}} v_{*} t}\right) \leqslant \frac{C}{L} e^{-2 \sqrt{\omega_{*}} v_{*} t} .
\end{aligned}
$$

Therefore, choosing $L$ large enough we obtain the required estimate (35). This concludes the proof of Proposition 5 .

\section{Numerical SCHEMES}

We describe here the numerical methods that we will be using in the next section.

4.1. The time-splitting spectral method. We start by the time-splitting spectral method that we use to solve (NLS) numerically. The equations are solved on a bounded interval $I=(-a, a)$. We use a uniform spatial grid with mesh size $h>0$ and grid points $x_{k}=x_{0}+k h, k=0, \ldots, K$, where $K+1 \in \mathbb{N}$ is the (odd) number of grid points. Then $h=2 a / K$. The time grid is given by $t_{n}=t_{0}+n \tau, n \in \mathbb{N}_{0}$, where $\tau>0$ is the time step size and $t_{0}$ the initial time. We set $\left(u_{j}\right)_{k}^{n}:=u_{j}\left(t_{n}, x_{k}\right)$, where $j=1,2, k=0, \ldots, K$, and $n \in \mathbb{N}_{0}$. We split the system (NLS) into the two subsystems

$$
\begin{aligned}
& i \partial_{t} u_{j}=-\mu_{j}\left|u_{j}\right|^{2} u_{j}-\beta\left|u_{3-j}\right|^{2} u_{j}, \quad j=1,2, \\
& i \partial_{t} u_{j}=-\frac{1}{2} \partial_{x x} u_{j}, \quad j=1,2,
\end{aligned}
$$

considered on $\left[t_{n}, t_{n+1}\right]$ and subject to some initial data. These subsystems are solved as follows.

Step 1: Computing the evolution of (36) we observe that the quantities $\left|u_{j}\right|^{2}$ remain unchanged. Therefore, we "freeze" these values at time $t_{n}$ and solve the resulting linear ODEs exactly in the interval $\left[t_{n}, t_{n}+\tau / 2\right]$, giving at time $t_{n}+\tau / 2$ :

$$
\left(u_{1}\right)_{k}^{*}=\exp \left(i \frac{\tau}{2}\left(\mu_{1}\left|\left(u_{1}\right)_{k}^{n}\right|^{2}+\beta\left|\left(u_{2}\right)_{k}^{n}\right|^{2}\right)\right)\left(u_{1}\right)_{k}^{n},
$$

and analogously for $\left(u_{2}\right)_{k}^{*}$.

Step 2: We solve (37) for $j=1,2$ in the interval $\left[t_{n}, t_{n}+\tau\right]$, discretized in space by the Fourier spectral method and solved exactly in time:

$$
\begin{aligned}
& \left(u_{j}\right)_{k}^{* *}=\frac{1}{K+1} \sum_{m=-K / 2}^{K / 2} \exp \left(-i \tau \nu_{m}^{2}\right)\left(\widehat{u}_{j}\right)_{m}^{*} \exp \left(i \nu_{m}\left(x_{k}-x_{0}\right)\right), \quad j=1,2, \\
& \text { where } \nu_{m}=2 \pi m /\left(x_{K}-x_{0}\right) \text { and } \\
& \left(\widehat{u}_{j}\right)_{m}^{*}=\sum_{l=0}^{K}\left(u_{j}\right)_{l}^{*} \exp \left(-i \nu_{m}\left(x_{l}-x_{0}\right)\right), \quad m=-\frac{K}{2}, \ldots, \frac{K}{2} .
\end{aligned}
$$

Step 3: We solve (36) on $\left[t_{n}+\tau / 2, t_{n+1}\right]$ using the discretization of Step 1 with $\left(u_{j}\right)_{k}^{* *}$ instead of $\left(u_{j}\right)_{k}^{n}$ and obtain $\left(u_{j}\right)_{k}^{n+1}$. 
4.2. The normalized gradient flow. We will also need to compute the ground state solution of the following elliptic system with fixed masses

$$
\left\{\begin{array}{l}
-\partial_{x x} \phi_{1}+\omega_{1} \phi_{1}-\mu_{1} \phi_{1}^{3}-\beta \phi_{2}^{2} \phi_{1}=0 \\
-\partial_{x x} \phi_{2}+\omega_{2} \phi_{2}-\mu_{2} \phi_{2}^{3}-\beta \phi_{1}^{2} \phi_{2}=0
\end{array}\right.
$$

To that purpose, we use the normalized gradient flow. The problem can also be viewed as a nonlinear eigenvalue problem with $\omega_{1}, \omega_{2}$ being the eigenvalues, which can be computed from the corresponding eigenfunctions $(j=1,2)$ :

$$
\omega_{j}^{\phi}=\frac{\int_{\mathbb{R}}\left(-\left|\partial_{x} \phi_{j}\right|^{2}+\mu_{j} \phi_{j}^{4}+\beta \phi_{1}^{2} \phi_{2}^{2}\right) d x}{\int_{\mathbb{R}} \phi_{j}^{2} d x} .
$$

We solve (38) by normalized gradient flow with given $\left(a_{1}, a_{2}\right)$, such that:

$$
\int_{\mathbb{R}} \phi_{1}^{2} d x=a_{1}^{2} \quad \text { and } \quad \int_{\mathbb{R}} \phi_{2}^{2} d x=a_{2}^{2} .
$$

The standard gradient flow with discrete normalization consists in introducing an imaginary time in the nonlinear Schrödinger equations, thus looking at the imaginary time propagation $(t \rightarrow-i t)$ and after every step project the solutions such that the $L^{2}$-norms are equal to $\left(a_{1}^{2}, a_{2}^{2}\right)$. In [6] the authors present the normalized gradient flow, prove it is energy diminishing, and propose numerical methods to discretize it. Hereafter we adapted the normalized gradient flow for the given system and discretized it by a semi-implicit Backward Euler finite differences scheme. For $\left(\phi_{j}\right)_{k}^{n}=\phi_{j}\left(t^{n}, x_{k}\right)$ being the discrete solution, $x_{k}=x_{0}+k \cdot h$ the grid points with $k=0,1, \ldots, K-1, K$ and the time sequence $0<t_{1}<t_{2}<\cdots<t_{n}<t_{n+1}<\ldots$ with $\tau=t_{n+1}-t_{n}$ we have the following discretization of the normalized gradient flow:

Step 1: We first solve on $\left[t_{n}, t_{n+1}\right]$ with initial data $\phi_{j}\left(t_{n}, x_{k}\right)$ :

$\frac{\left(\phi_{j}\right)_{k}^{*}-\left(\phi_{j}\right)_{k}^{n}}{\tau}=\frac{\left(\phi_{j}\right)_{k+1}^{*}-2\left(\phi_{j}\right)_{k}^{*}+\left(\phi_{j}\right)_{k-1}^{*}}{h^{2}}+\mu_{j}\left|\left(\phi_{j}\right)_{k}^{n}\right|^{2}\left(\phi_{j}\right)_{k}^{*}+\beta\left|\left(\phi_{3-j}\right)_{k}^{n}\right|^{2}\left(\phi_{j}\right)_{k}^{*}$ with $j=\{1,2\}$. As solution we get $\left(\phi_{j}\right)_{k}^{*}$.

Step 2: $\left(\phi_{j}\right)_{k}^{*}$ is then normalized to get finally $\left(\phi_{j}\right)_{k}^{n+1}$

$$
\left(\phi_{j}\right)_{k}^{n+1}=\frac{a_{k}\left(\phi_{j}\right)_{k}^{*}}{\left\|\left(\phi_{j}\right)_{k}^{*}\right\|_{L^{2}}} .
$$

For $t \rightarrow+\infty$ we obtain the ground state solution $\left(\phi_{1}, \phi_{2}\right)$ of (38) with $L^{2}$-norms equal to $\left(a_{1}^{2}, a_{2}^{2}\right)$ and frequency parameters $\left(\omega_{1}^{\phi_{1}}, \omega_{2}^{\phi_{2}}\right)$.

\section{NUMERICAL EXPERIMENTS}

Our ansatz for the initial data in the next experiments is the following.

$$
u_{j}\left(t_{0}, x\right)=e^{i\left(\omega_{j} t_{0}-v_{j}^{2} t / 4+v_{j} x / 2\right)} Q_{\omega_{j}}\left(x-v_{j} t_{0}-x_{j}\right) \quad j=1,2
$$

with $Q_{\omega}$ defined in (3). Without loss of generality, we may assume that $0<v_{1}=$ $-v_{2}$ (Galilean invariance), hence the soliton on the first component will be traveling to the right and the soliton on the second component will be traveling to the left. We will also assume that $x_{1}=x_{2}=0$ (invariance by translation in time and space) and to guarantee that our solitons on the first and second components are positioned 
on the left and on the right respectively and at a sufficiently large distance, we will chose the initial time to be $t_{0}=-10$.

5.1. Purely elastic interaction. The integrable case has been studied in depth in the book 1. In particular, in that case the system is completely integrable via the inverse scattering transform and explicit solutions may be exhibited. For example, there exists a solution $U=\left(u_{1}, u_{2}\right)$ of (NLS) which has the following behavior. At time $t \rightarrow-\infty$,

$$
\begin{aligned}
& u_{1}(t, x) \sim e^{i\left(\frac{1}{2} v_{1} x+\left(\omega_{1}-\frac{1}{4}\left|v_{1}\right|^{2}\right) t\right)} \sqrt{\omega_{1}} Q\left(\sqrt{\omega_{1}}\left(x-v_{1} t\right)\right), \\
& u_{2}(t, x) \sim e^{i\left(\frac{1}{2} v_{2} x+\left(\omega_{2}-\frac{1}{4}\left|v_{2}\right|^{2}\right) t\right)} \sqrt{\omega_{2}} Q\left(\sqrt{\omega_{2}}\left(x-v_{2} t\right)\right),
\end{aligned}
$$

whereas at time $t \rightarrow+\infty$ the two components have interacted and the outcome are solitons of same speed and frequency but with a shift in phase and translation:

$$
\begin{aligned}
& u_{1}(t, x) \sim \hat{\phi}_{1}(t, x):=e^{i \theta_{1}} e^{i\left(\frac{1}{2} v_{1} x+\left(\omega_{1}-\frac{1}{4}\left|v_{1}\right|^{2}\right) t\right)} \sqrt{\omega_{1}} Q\left(\sqrt{\omega_{1}}\left(x-v_{1} t-\tau_{1}\right)\right), \\
& u_{2}(t, x) \sim \hat{\phi}_{2}(t, x):=e^{i \theta_{2}} e^{i\left(\frac{1}{2} v_{2} x+\left(\omega_{2}-\frac{1}{4}\left|v_{2}\right|^{2}\right) t\right)} \sqrt{\omega_{2}} Q\left(\sqrt{\omega_{2}}\left(x-v_{2} t-\tau_{2}\right)\right),
\end{aligned}
$$

where the shift parameters are given by the formulas

$$
\begin{array}{r}
\tau_{1}=-\frac{\ln \left(\left|\chi_{1}\right|\right)}{\sqrt{\omega_{1}}}, \quad \tau_{2}=\frac{\ln \left(\left|\chi_{2}\right|\right)}{\sqrt{\omega_{2}}}, \quad \theta_{1}=\frac{\chi_{1}}{\left|\chi_{1}\right|}, \quad \theta_{2}=\frac{\chi_{2}}{\left|\chi_{2}\right|}, \\
\chi_{1}=\frac{v_{1}-v_{2}+i 2\left(\sqrt{\omega_{1}}+\sqrt{\omega_{2}}\right)}{v_{1}-v_{2}+i 2\left(\sqrt{\omega_{1}}-\sqrt{\omega_{2}}\right)}, \quad \chi_{2}=\frac{v_{1}-v_{2}+i 2\left(\sqrt{\omega_{1}}+\sqrt{\omega_{2}}\right)}{v_{1}-v_{2}-i 2\left(\sqrt{\omega_{1}}-\sqrt{\omega_{2}}\right)} .
\end{array}
$$

Note that since the speeds and amplitudes of the solitons are not modified the interaction is considered elastic. Note also that we have a pure two-speeds solitary wave at both ends of the time line, without any appearance of dispersion despite the interaction. This is a characteristic feature of completely integrable systems.

The parameters are chosen as follows: $\mu_{1}=\mu_{2}=\beta=1, \omega_{1}=5, \omega_{2}=1$, $v_{1}=1, v_{2}=-1$, and $x_{1}=x_{2}=0$, thus we start with two solitons located at \pm 10 , respectively, which move to each other and observe them until $t_{\text {final }}=10$. Furthermore, the integration domain is $I=(-20,20)$ with $K=1024$ spatial grid points and $\tau=10^{-3}$.
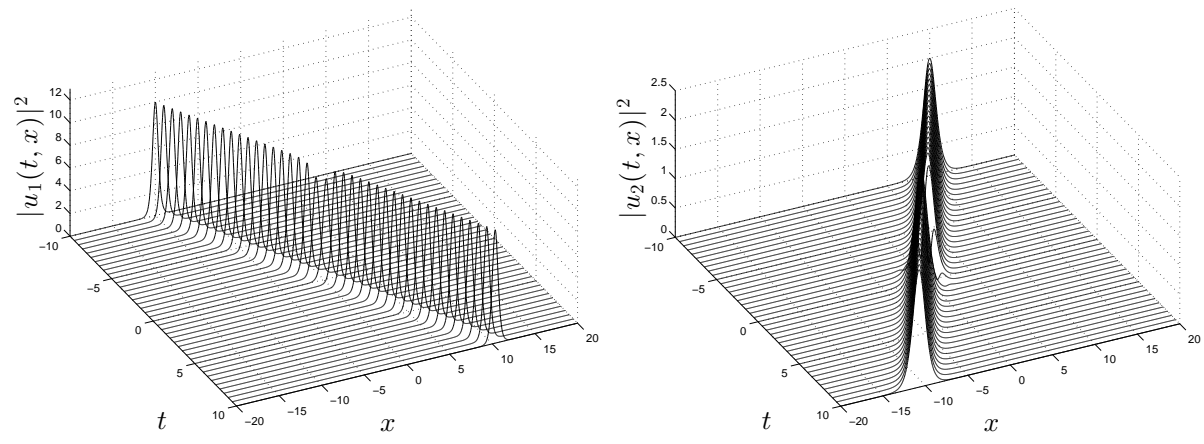

Figure 1. Purely elastic interaction: Position densities $\left|u_{1}(t, x)\right|^{2}$ and $\left|u_{2}(t, x)\right|^{2}$ as functions of space and time for $\mu_{1}=$ $\mu_{2}=1$ and $\beta=1$ 
We plot the solution $\left(u_{1}, u_{2}\right)$ to (NLS) and observe that the two solitons remain unchanged after the interaction, excepting a shift in phase and translation. The shift in translation can be observed in Figure 1. Our numerical experiments are in good line with the theoretically predicted behavior. We have compared pointwise the numerical solution $\left|u_{1}\right|^{2}$ obtained by taking (40) as initial data at $t=-10$ and the theoretical outcome $\left|\hat{\phi}_{1}\right|^{2}$ given by (41). The results are shown in Figure 2 .

a)

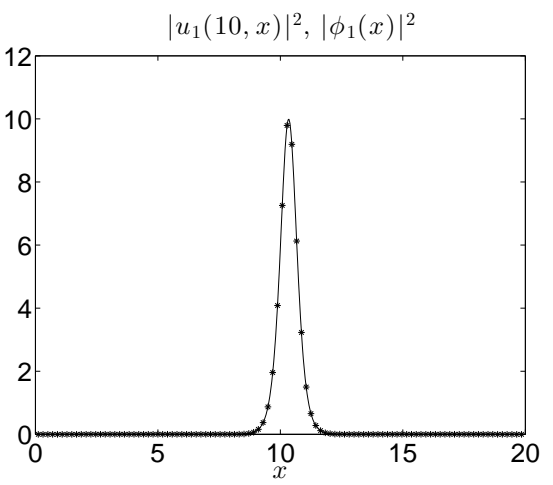

b)

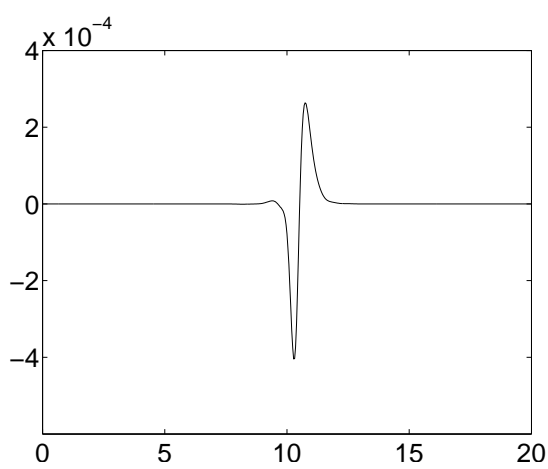

Figure 2. a) Plots of $\left|u_{1}(10, x)\right|^{2}$ ('-' line) and $\left|\hat{\phi}_{1}(x)\right|^{2}$ ('.' line).

b) Difference of $\left|u_{1}(10, x)\right|^{2}-\left|\hat{\phi}_{1}(x)\right|^{2}$

5.2. Symmetric collision. In this experiment the outcome are still multi-speed solitary waves. After the collision we observe a small part of the soliton moving along with the soliton of the other component. We see that at the final time the solution fits well the ground state solution obtained by minimizing the system with fixed masses. Here we choose $\mu_{1}=\mu_{2}=1$ and $\beta=3$. We have solved the system (NLS) in the interval $(-200,200)$ with periodic boundary conditions, initial time $t_{0}=-10$ and initial condition (39) and the parameters $\omega_{1}=\omega_{2}=1$, $v_{1}=2, v_{2}=-2$, and $x_{1}=x_{2}=0$, thus we start with two solitons located at \pm 10 , respectively, which move to each other and observe them till $t_{\text {final }}=40$. Moreover, we use $K=4096$ in space and time step $\tau=10^{-3}$.

Here the interaction parameter $\beta$ is greater than in the first experiment and after the interaction at time zero we see in each component a small part going with the other component, respectively (Figure 3).

At the final time $t=40$ we look at the left side part of each component $u_{j}^{-}=$ $u_{j} \cdot \chi_{[-\infty, 0]}$, and compute the $L^{2}$-norms

$$
a_{j}^{2}=\int_{\mathbb{R}}\left|u_{j}^{-}(40, x)\right|^{2} d x .
$$

Note that the mass of each component $M_{j}(t)=\frac{1}{2} \int_{\mathbb{R}}\left|u_{j}(t, x)\right|^{2} d x$ is conserved, $M_{j}(0)=M_{j}(t)$ for $j=1,2$ and for all $t \in[-10,40]$. At $t=40$ we obtain $\left(a_{1}=\right.$ $\sqrt{3.893}, a_{2}=\sqrt{0.069}$.

Finally we compute the ground state solution $\left(\phi_{1}(x), \phi_{2}(x)\right)$ of the elliptic system (38) by the gradient flow with constraints $(3.893,0.069)$ and compare it to $\left(u_{1}^{-}(40, x), u_{2}^{-}(40, x)\right)$. In Figure 4 we first shift $\phi_{1}\left(x+x_{1}\right)$ (with $x_{1}$ corresponding to $\max \left|u_{1}\right|^{2}$ ) and $\phi_{2}\left(x+x_{2}\right)$ (with $x_{2}$ corresponding to $\max \left|u_{2}\right|^{2}$ ) and then compare 

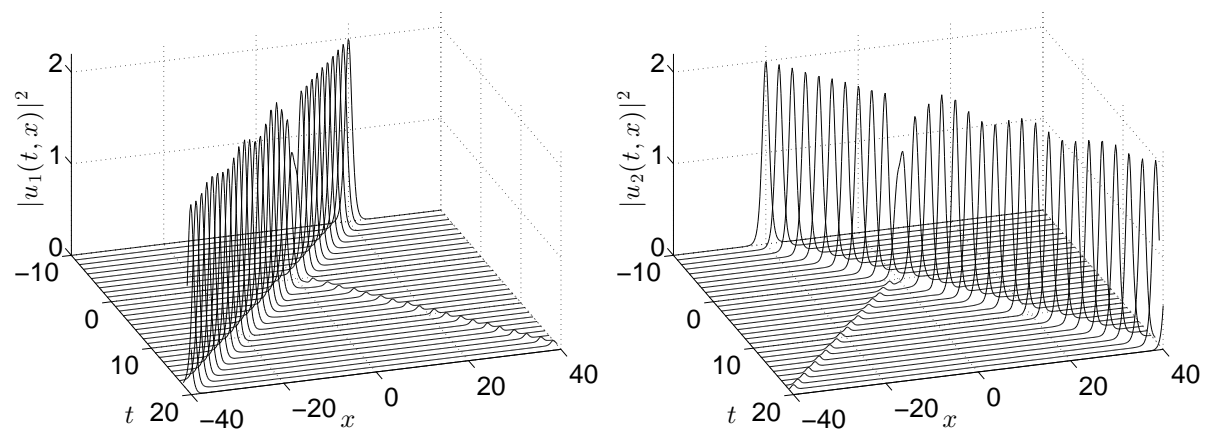

FIgURE 3. Symmetric collision: Position densities $\left|u_{1}(t, x)\right|^{2}$ and $\left|u_{2}(t, x)\right|^{2}$ as functions of space and time for $\mu_{1}=\mu_{2}=1$ and $\beta=3$

a)

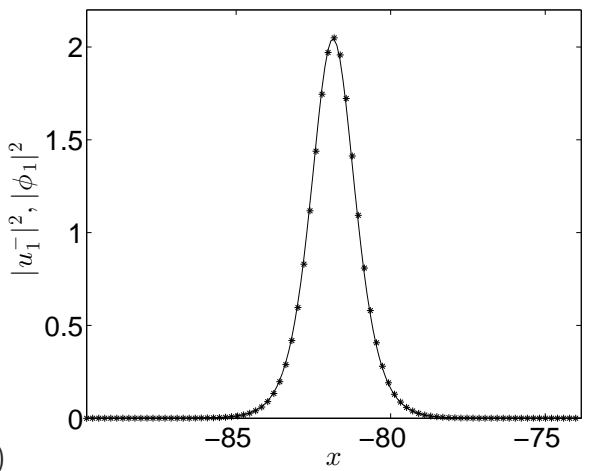

b)

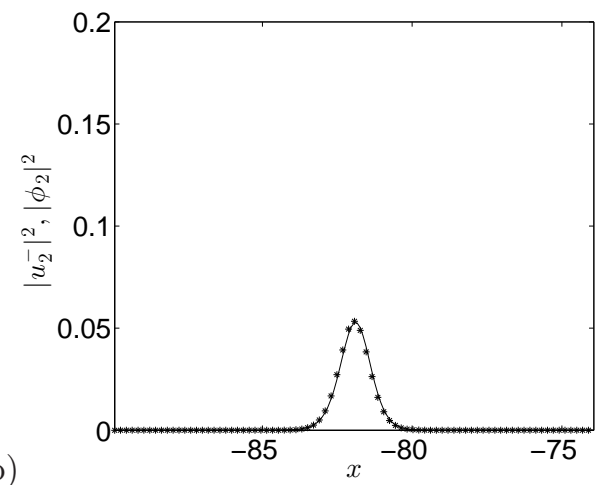

Figure 4. Symmetric collision: a) Plots of $\left|u_{1}^{-}(40, x)\right|^{2}$ ('' line) and $\left|\phi_{1}\left(x+x_{1}\right)\right|^{2}$ ('.' line) b) Plots of $\left|u_{2}^{-}(40, x)\right|^{2}$ and $\left|\phi_{2}\left(x+x_{2}\right)\right|^{2}$, where $x_{j}$ is the $x$ corresponding to the $\max \left|u_{j}\right|^{2}$, with $j=1,2$.

the position density pointwise to the position density of the final solution located on the left of the origin $\left(u_{1}^{-}(40, x), u_{2}^{-}(40, x)\right)$ and realize that they fit very well.

In this case, the interaction result into a new repartition of the mass and of the energy so as to approximate a ground state profile.

5.3. Dispersive inelastic interaction. In this experiment we observe a loss of energy, mass and momentum in a small dispersive part located at the interaction place and a small dispersive part moving to the boundaries. Here we have solved the system (NLS) with $\beta=-1$ and $\mu_{1}=\mu_{2}=1$ in the interval $(-500,500)$ with 8192 grid points for the spatial discretization, with periodic boundary conditions, initial time $t_{0}=-10$ and initial condition (39) with the following parameters $v_{1}=-v_{2}=2.7, \omega_{1}=\omega_{2}=1$, and $x_{1}=x_{2}=0$.

Figure 5 shows the evolution of the waves. In Figure 6 we see the position density of the initial soliton $u_{1}(0, x)$ and the one of the final soliton at $t=90$, with lost mass and energy. 

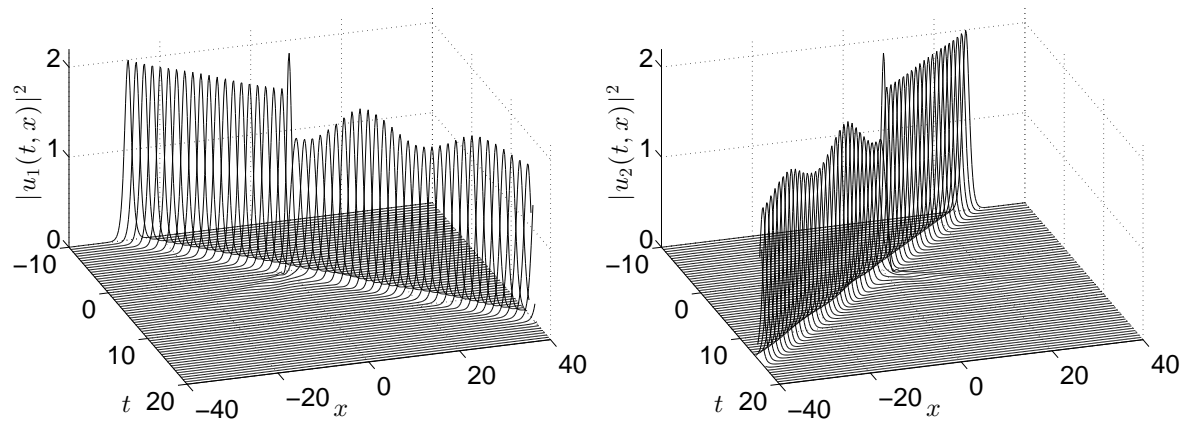

Figure 5. Dispersive inelastic interaction: Plots of $\left|u_{1}(t, x)\right|^{2}$ (left) and $\left|u_{2}(t, x)\right|^{2}$ (right) as functions of space and time
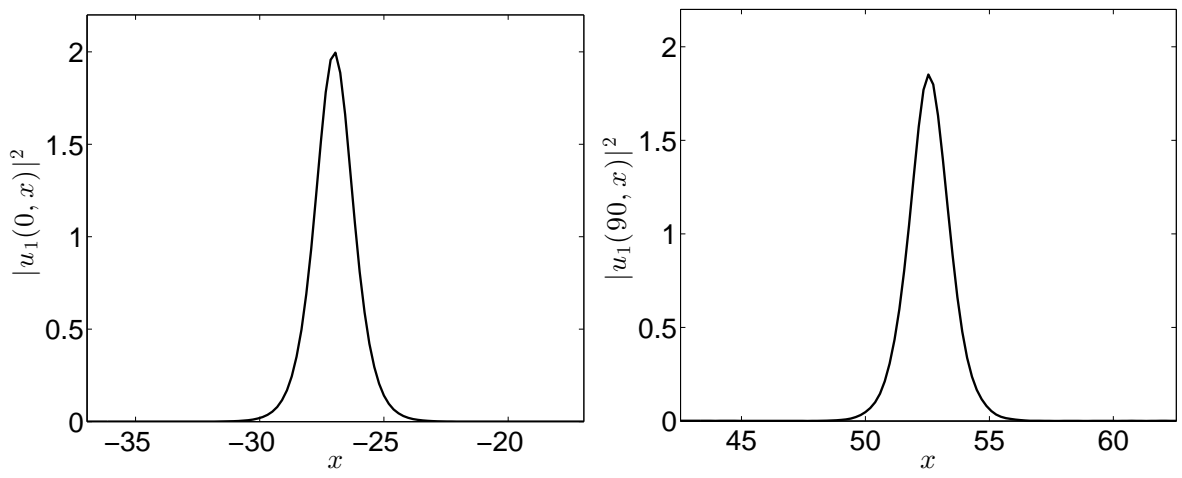

\section{Figure 6. Dispersive inelastic interaction: Plots of $\left|u_{1}(0, x)\right|^{2}$ (left) and $\left|u_{1}(90, x)\right|^{2}$ (right)}

5.4. Reflexion. In this experiment we observe a change of the sign of the speeds, thus the solitons are reflected after interaction. Here we have the same parameter as in the case of a dispersive interaction, $\beta=-1$ and $\mu_{1}=\mu_{2}=1$, but the initial velocity is smaller, precisely we have the initial condition (39) with the following parameters $v_{1}=-v_{2}=0.5, \omega_{1}=\omega_{2}=1$, and $x_{1}=x_{2}=0$. Since the velocity is not so large, and we observe the soliton until $t_{\text {final }}=10$, we solve (NLS) on $(-20,20)$ with 1024 spatial grid points, periodic boundary conditions and time step equal to $\tau=10^{-3}$.

In Figure 7 we see the reflexion of the two solitons after interaction, thus only the velocities changed sign. In Figure 8 we compare point wise the solution at the final time to the initial solution, and observe that it remains unchanged.

These were various examples of the possible outcomes of multi-speeds solitary waves interactions. The above described situations may not be the only possibles and we do not claim comprehensiveness. 

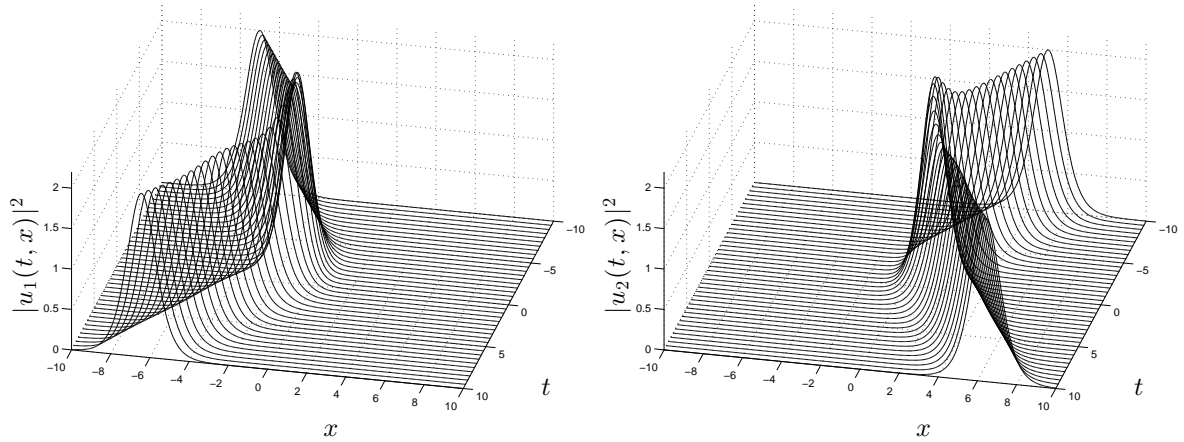

Figure 7. Reflexion: Plots of $\left|u_{1}(t, x)\right|^{2}$ (left) and $\left|u_{2}(t, x)\right|^{2}$ (right) as functions of space and time

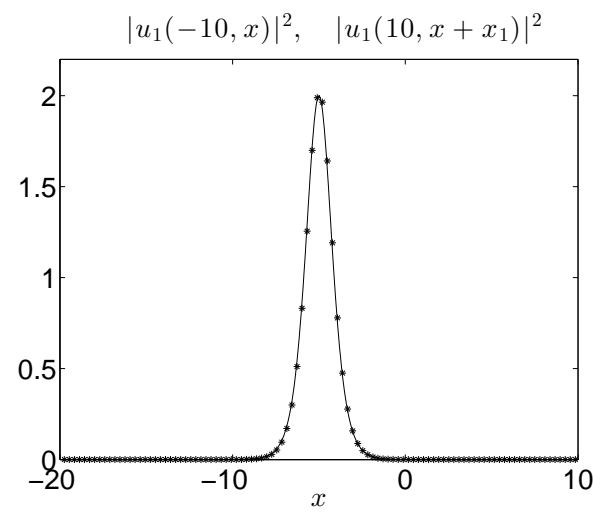

Figure 8. Reflexion: Plots of $\left|u_{1}\left(-10, x-x_{1}\right)\right|^{2}$ ('-' line) and $\left|u_{1}(10, x)\right|^{2}$ ('.$'$ line), where $x_{1}$ is the translation such that we can compare the two solitons

\section{Appendix A. Proof of the modulation lemma}

Proof of Lemma 6. The proof of the modulation lemma is inspired from [23] and relies on the implicit function theorem.

Let us define $F: H^{1}(\mathbb{R}) \times \mathbb{R}_{+} \times \mathbb{R} \times \mathbb{R} \longrightarrow \mathbb{R}^{3}$ by

$$
F(u, \omega, \gamma, y)=\left(\begin{array}{c}
(u-R(\omega, \gamma, y), R(\omega, \gamma, y))_{2} \\
(u-R(\omega, \gamma, y), i R(\omega, \gamma, y))_{2} \\
\left(u-R(\omega, \gamma, y), \partial_{x} R(\omega, \gamma, y)\right)_{2}
\end{array}\right)
$$

where, for the sake of simplicity, we removed the $i$ indexes and $R(\omega, \gamma, y)$ denotes the soliton $R(\omega, \gamma, y)=e^{i\left(\frac{1}{2} v \cdot x+\gamma\right)} \frac{1}{\sqrt{\mu}} Q_{\omega}(x-y)$. Note that,

$$
\forall(\omega, \gamma, y) \in \mathbb{R}_{+} \times \mathbb{R} \times \mathbb{R}, \quad F(R(\omega, \gamma, y), \omega, \gamma, y)=0 .
$$

The key idea here is to apply the implicit function theorem to function $F$ at point $(R(\omega, \gamma, y), \omega, \gamma, y)$ for fixed $(\omega, \gamma, y) \in \mathbb{R}_{+} \times \mathbb{R} \times \mathbb{R}$. Simple computations due to properties of $Q_{\omega}$, mainly the fact that $Q_{\omega}$ is radial and even, lead to 
$\nabla F(R(\omega, \gamma, y), \omega, \gamma, y)$ diagonal with diagonal terms $a, b, c$ given by:

$$
\begin{aligned}
a & =\frac{1}{\mu} \mathcal{R} e \int_{\mathbb{R}} \partial_{\omega} Q_{\omega}(x-y) \overline{Q_{\omega}(x-y)} d x<0 \\
b & =-\left(\partial_{\gamma} R(\omega, \gamma, y), i R(\omega, \gamma, y)\right)_{2}=\frac{-1}{\mu} \mathcal{R} e \int_{\mathbb{R}} Q_{\omega}(x-y)^{2} d x<0 \\
c & =\frac{-1}{\mu}\left\|\partial_{k} Q_{\omega}\right\|_{L^{2}}^{2}<0 .
\end{aligned}
$$

Implicit function theorem finally gives the existence of parameters $\tilde{\omega}_{j}, \tilde{\gamma}_{j}, \tilde{x}_{j}$ as functions of time. To prove that these functions are actually of class $\mathcal{C}^{1}$, a standard regularization argument is needed. We refer to 22 for more details on that argument.

Now, in order to be more readable, we prove estimate (13) for $j=1$. In that purpose, let us write the equation of evolution satisfied by $\varepsilon_{1}$, namely:

$$
\begin{aligned}
& i \partial_{t} \varepsilon_{1}+L\left(\varepsilon_{1}, \varepsilon_{2}\right)+\mathcal{N}\left(\varepsilon_{1}, \varepsilon_{2}\right) \\
& \quad=-\left(\tilde{\omega}_{1}-\frac{v_{1}^{2}}{4}-\partial_{t} \tilde{\gamma}_{1}\right) \tilde{R}_{1}-i \partial_{t} \tilde{\omega}_{1} \partial_{\omega} Q_{\tilde{\omega}_{1}} e^{i\left(\frac{1}{2} v_{1} \cdot x+\tilde{\gamma}_{1}\right)}-i\left(v_{1}-\partial_{t} \tilde{x}_{1}\right) \partial_{x} Q_{\tilde{\omega}_{1}} .
\end{aligned}
$$

where

$$
L\left(\varepsilon_{1}, \varepsilon_{2}\right)=\partial_{x x} \varepsilon_{1}+\left(\mu_{1}\left|\tilde{R}_{1}\right|^{2}+\beta\left|\tilde{R}_{2}\right|^{2}\right) \varepsilon_{1}+2 \mu_{1} \operatorname{Re}\left(\tilde{R}_{1} \overline{\varepsilon_{1}}\right) \tilde{R}_{1}+2 \beta \mathcal{R} e\left(\tilde{R}_{2} \bar{\varepsilon}_{2}\right) \tilde{R}_{1}
$$

and

$$
\begin{aligned}
\mathcal{N}\left(\varepsilon_{1}, \varepsilon_{2}\right)=\mu_{1}\left|\varepsilon_{1}\right|^{2} \varepsilon_{1}+\beta\left|\varepsilon_{2}\right|^{2} \varepsilon_{1}+\left(\mu_{1}\left|\varepsilon_{1}\right|^{2}\right. & \left.+\beta\left|\varepsilon_{2}\right|^{2}\right) \tilde{R}_{1} \\
& +\left(2 \mu_{1} \operatorname{Re}\left(\tilde{R}_{1} \varepsilon_{1}\right)+2 \beta \mathcal{R} e\left(\tilde{R}_{2} \overline{\varepsilon_{2}}\right)\right) \varepsilon_{1} .
\end{aligned}
$$

It is to be noticed that the modulation terms $\tilde{\omega}_{1}-\frac{v_{1}^{2}}{4}-\partial_{t} \tilde{\gamma}_{1}, \partial_{t} \tilde{\omega}_{1}$, and $v_{1}-\partial_{t} \tilde{x}_{1}$ appear in the right hand side of this evolution equation. The main idea of the proof is to take in (42) the scalar products of both sides of the equation with respectively $\tilde{R}_{1}, i \tilde{R}_{1}$ and $\partial_{x} \tilde{R}_{1}$. We then use the orthogonality conditions (12) as well as properties on $Q_{\tilde{\omega}_{1}}$. In the left hand side, we transfer the derivatives acting on $\varepsilon_{1}$ on the other side of the scalar product thanks to the modulation conditions (12) (for the time derivatives) and integrations by parts (for the space derivatives). We finally use the equation at hand on $\tilde{R}_{1}$ :

$$
\begin{aligned}
& i \partial_{t} \tilde{R}_{1}+\partial_{x x} \tilde{R}_{1}+\mu_{1}\left|\tilde{R}_{1}\right|^{2} \tilde{R}_{1}= \\
& \left(\tilde{\omega}_{1}+\frac{v_{1}^{2}}{4}-\partial_{t} \tilde{\gamma}_{1}\right) \tilde{R}_{1}+\frac{j}{\sqrt{\mu_{1}}} e^{i\left(\frac{1}{2} v_{1} \cdot x+\tilde{\gamma}_{1}\right)} \partial_{t} \tilde{\omega}_{1} \partial_{\omega} Q_{\tilde{\omega}_{1}}+i \frac{v_{1}-\partial_{t} \tilde{x}_{1}}{\mu_{1}} e^{i\left(\frac{1}{2} v_{1} \cdot x+\tilde{\gamma}_{1}\right)} \partial_{x} Q_{\tilde{\omega}_{1}}
\end{aligned}
$$

For more simplicity, we only develop the computations for the scalar product with $\tilde{R}_{1}$, the other cases are obtained using the same arguments. 
Taking the scalar product with $\tilde{R}_{1}$ in equation (42) leads to:

$$
\begin{aligned}
&\left(i \partial_{t} \varepsilon_{1}+L\left(\varepsilon_{1}, \varepsilon_{2}\right)+\right.\left.\mathcal{N}\left(\varepsilon_{1}, \varepsilon_{2}\right), \tilde{R}_{1}\right)_{2}=-\left(\tilde{\omega}_{1}-\frac{v_{1}^{2}}{4}-\partial_{t} \tilde{\gamma}_{1}\right)\left\|\tilde{R}_{1}\right\|^{2} \\
&-\partial_{t} \tilde{\omega}_{1} \operatorname{Re} \int_{\mathbb{R}} i e^{i\left(\frac{1}{2} v_{1} \cdot x+\tilde{\gamma}_{1}\right)} \partial_{\omega} Q_{\tilde{\omega}_{1}} \overline{\tilde{R}_{1}} d x \\
& \quad-\left(v_{1}-\partial_{t} \tilde{x}_{1}\right) \operatorname{Re} \int_{\mathbb{R}} i e^{i\left(\frac{1}{2} v_{1} \cdot x+\tilde{\gamma}_{1}\right)} \partial_{x} Q_{\tilde{\omega}_{1}} \overline{\tilde{R}_{1}} d x
\end{aligned}
$$

Right hand side of (44): First, using equation (11) leads to:

$$
\mathcal{R e} \int_{\mathbb{R}} i e^{i\left(\frac{1}{2} v_{1} \cdot x+\tilde{\gamma}_{1}\right)} \partial_{\omega} Q_{\tilde{\omega}_{1}} \overline{\tilde{R}_{1}} d x=\frac{1}{\sqrt{\mu_{1}}} \mathcal{I}_{m} \int_{\mathbb{R}} \partial_{\omega} Q_{\tilde{\omega}_{1}}(x) Q_{\tilde{\omega}_{1}}\left(x-\tilde{x}_{1}\right) d x=0
$$

and

$$
\mathcal{R e} \int_{\mathbb{R}} i e^{i\left(\frac{1}{2} v_{1} \cdot x+\tilde{\gamma}_{1}\right)} \partial_{x} Q_{\tilde{\omega}_{1}} \overline{\tilde{R}_{1}} d x=\frac{1}{\sqrt{\mu_{1}}} \mathcal{I}_{m} \int_{\mathbb{R}} \partial_{x} Q_{\tilde{\omega}_{1}}(x) Q_{\tilde{\omega}_{1}}\left(x-\tilde{x}_{1}\right) d x=0 .
$$

Thus, (44) reduces to:

$$
\left(i \partial_{t} \varepsilon_{1}+L_{1}\left(\varepsilon_{1}, \varepsilon_{2}\right)+\mathcal{N}\left(\varepsilon_{1}, \varepsilon_{2}\right), \tilde{R}_{1}\right)_{2}=-\left(\tilde{\omega}_{1}-\frac{v_{1}^{2}}{4}-\partial_{t} \tilde{\gamma}_{1}\right)\left\|\tilde{R}_{1}\right\|^{2} .
$$

Left hand side of (45):

First, deriving modulation condition $\left(\varepsilon_{1}, \tilde{R}_{1}\right)_{2}=0$ with respect to time gives: $\left(i \partial_{t} \varepsilon_{1}, \tilde{R}_{1}\right)_{2}=\left(\varepsilon_{1}, i \partial_{t} \tilde{R}_{1}\right)_{2}$.

Let us now develop $\left(L\left(\varepsilon_{1}, \varepsilon_{2}\right), \tilde{R}_{1}\right)_{2}$ :

$$
\left(L\left(\varepsilon_{1}, \varepsilon_{2}\right), \tilde{R}_{1}\right)_{2}=\left(\varepsilon_{1}, \partial_{x x} \tilde{R}_{1}+3 \mu_{1}\left|\tilde{R}_{1}\right|^{2} \tilde{R}_{1}+\beta\left|\tilde{R}_{2}\right|^{2} \tilde{R}_{1}\right)_{2}+2 \beta\left(\varepsilon_{2},\left|\tilde{R}_{1}\right|^{2} \tilde{R}_{2}\right)_{2} .
$$

Finally, $\left(\mathcal{N}\left(\varepsilon_{1}, \varepsilon_{2}\right), \tilde{R}_{1}\right)_{2}$ read:

$$
\begin{array}{r}
\left(\mathcal{N}\left(\varepsilon_{1}, \varepsilon_{2}\right), \tilde{R}_{1}\right)_{2}=\left(\mu_{1}\left|\varepsilon_{1}\right|^{2} \varepsilon_{1}+\beta\left|\varepsilon_{2}\right|^{2} \varepsilon_{1}, \tilde{R}_{1}\right)_{2}+\left(\left(\mu_{1}\left|\varepsilon_{1}\right|^{2}+\beta\left|\varepsilon_{2}\right|^{2}\right) \tilde{R}_{1}, \tilde{R}_{1}\right)_{2} \\
+\left(\left(2 \mu_{1} \operatorname{Re}\left(\tilde{R}_{1} \varepsilon_{1}\right)+2 \beta \mathcal{R}\left(\tilde{R}_{2} \overline{\varepsilon_{2}}\right)\right) \varepsilon_{1}, \tilde{R}_{1}\right)_{2}
\end{array}
$$

Equation (45) thus leads to the left hand side term:

$$
\begin{aligned}
\left(\varepsilon_{1}, i \partial_{t} \tilde{R}_{1}+\partial_{x x} \tilde{R}_{1}+\mu_{1}\left|\tilde{R}_{1}\right|^{2} \tilde{R}_{1}\right)_{2}+\beta\left(\varepsilon_{1},\left|\tilde{R}_{2}\right|^{2} \tilde{R}_{1}\right)_{2}+2 \mu_{1}\left(\varepsilon_{1},\left|\tilde{R}_{1}\right|^{2} \tilde{R}_{1}\right)_{2} \\
+2 \beta\left(\varepsilon_{2},\left|\tilde{R}_{1}\right|^{2} \tilde{R}_{2}\right)_{2}+\left(\mathcal{N}\left(\varepsilon_{1}, \varepsilon_{2}\right), \tilde{R}_{1}\right)_{2} .
\end{aligned}
$$

Now, using equation (43) satisfied by $\tilde{R}_{1}$ gives:

$$
\begin{aligned}
& \left(\varepsilon_{1}, i \partial_{t} \tilde{R}_{1}+\partial_{x x} \tilde{R}_{1}+\mu_{1}\left|\tilde{R}_{1}\right|^{2} \tilde{R}_{1}\right)_{2} \\
& =\left(\tilde{\omega}_{1}-\frac{v_{1}^{2}}{4}-\partial_{t} \tilde{\gamma}_{1}\right)\left(\varepsilon_{1}, \tilde{R}_{1}\right)_{2}+\partial_{t} \tilde{\omega}_{1}\left(\varepsilon_{1}, \frac{i}{\sqrt{\mu_{1}}} e^{i\left(\frac{1}{2} v_{1} \cdot x+\tilde{\gamma}_{1}\right)} \partial_{\omega} Q_{\tilde{\omega}_{1}}\right)_{2} \\
& \quad+\left(v_{1}-\partial_{t} \tilde{x}_{1}\right)\left(\varepsilon_{1}, \frac{i}{\sqrt{\mu_{1}}} e^{i\left(\frac{1}{2} v_{1} \cdot x+\tilde{\gamma}_{1}\right)} \partial_{x} Q_{\tilde{\omega}_{1}}\right)_{2}
\end{aligned}
$$


Finally, modulation condition $\left(\varepsilon_{1}, \tilde{R}_{1}\right)_{2}=0$ leads to

$$
\begin{gathered}
\left(\tilde{\omega}_{1}-\frac{v_{1}^{2}}{4}-\partial_{t} \tilde{\gamma}_{1}\right)\left\|\tilde{R}_{1}\right\|^{2}+\partial_{t} \tilde{\omega}_{1}\left(\varepsilon_{1}, \frac{i}{\sqrt{\mu_{1}}} e^{i\left(\frac{1}{2} v_{1} \cdot x+\tilde{\gamma}_{1}\right)} \partial_{\omega} Q_{\tilde{\omega}_{1}}\right)_{2} \\
+\left(v_{1}-\partial_{t} \tilde{x}_{1}\right)\left(\varepsilon_{1}, \frac{i}{\sqrt{\mu_{1}}} e^{i\left(\frac{1}{2} v_{1} \cdot x+\tilde{\gamma}_{1}\right)} \partial_{x} Q_{\tilde{\omega}_{1}}\right)_{2} \\
=-2 \mu_{1}\left(\varepsilon_{1},\left|\tilde{R}_{1}\right|^{2} \tilde{R}_{1}\right)_{2}-\beta\left(\varepsilon_{1},\left|\tilde{R}_{2}\right|^{2} \tilde{R}_{1}\right)_{2}-2 \beta\left(\varepsilon_{2},\left|\tilde{R}_{1}\right|^{2} \tilde{R}_{2}\right)_{2}-\left(\mathcal{N}\left(\varepsilon_{1}, \varepsilon_{2}\right), \tilde{R}_{1}\right)_{2}
\end{gathered}
$$

Thanks to Lemma 8 it is readily seen that most terms in this equation are of order $\mathcal{O}\left(\|\varepsilon\|_{2}\right)$. Finally, (46) can be re-written in a simpler way:

$$
\left(\left\|\tilde{R}_{1}\right\|^{2}+a_{1}(t)\right)\left(\tilde{\omega}_{1}-\frac{v_{1}^{2}}{4}-\partial_{t} \tilde{\gamma}_{1}\right)+a_{2}(t) \partial_{t} \tilde{\omega}_{1}+a_{3}(t)\left(v_{1}-\partial_{t} \tilde{x}_{1}\right)=b_{1}(t)
$$

where, for all $t \in\left[t_{0}, T^{n}\right],\left|a_{1}(t)\right|+\left|a_{2}(t)\right|+\left|a_{3}(t)\right|+\left|b_{1}(t)\right| \leqslant C\|\varepsilon(t)\|_{2}$.

With the same kind of arguments, taking in (42) scalar product with $i \tilde{R}_{1}$ and $i \partial_{x} \tilde{R}_{1}$ respectively, we get two other equations that can be re-written as a linear system solved by the "modulation vector" $\operatorname{Mod}(t)=\left(\begin{array}{c}\tilde{\omega}_{1}(t)-\frac{v_{1}^{2}}{4}-\partial_{t} \tilde{\gamma}_{1}(t) \\ \partial_{t} \tilde{\omega}_{1}(t) \\ v_{1}-\partial_{t} \tilde{x}_{1}(t) . .\end{array}\right)$ This linear system takes the following form:

$$
(\Gamma(t)+A(t)) \operatorname{Mod}(t)=B(t)
$$

where

$$
\Gamma(t)=\left(\begin{array}{ccc}
\left\|\tilde{R}_{1}\right\| & 0 & 0 \\
0 & \int_{\mathbb{R}} \partial_{\omega} Q_{\tilde{\omega}_{1}}(x) Q_{\tilde{\omega}_{1}}\left(x-\tilde{x}_{1}(t)\right) d x & 0 \\
\frac{v_{1}}{2}\left\|\tilde{R}_{1}\right\| & 0 & -\left\|\partial_{x} Q_{\tilde{\omega}_{1}}\right\|_{2}^{2}
\end{array}\right)
$$

and, with the help of Lemma 8 , for all $t \in\left[t_{0}, T^{n}\right],\|A(t)\|,\|B(t)\| \leqslant C\|\varepsilon\|_{2}$, and hence (13).

\section{REFERENCES}

[1] M. J. Ablowitz, B. Prinari, and A. D. Trubatch. Discrete and continuous nonlinear Schrödinger systems, volume 302 of London Mathematical Society Lecture Note Series. Cambridge University Press, Cambridge, 2004.

[2] G. Agrawal. Nonlinear fiber optics. Optics and Photonics. Academic Press, 2007.

[3] A. Ambrosetti and E. Colorado. Standing waves of some coupled nonlinear Schrödinger equations. J. Lond. Math. Soc. (2), 75(1):67-82, 2007.

[4] X. Antoine, W. Bao, and C. Besse. Computational methods for the dynamics of the nonlinear Schrödinger/Gross-Pitaevskii equations. Comput. Phys. Commun., 184(12):2621-2633, 2013.

[5] W. Bao. Ground states and dynamics of multicomponent Bose-Einstein condensates. Multiscale Model. Simul., 2:210-236, 2004.

[6] W. Bao and Q. Du. Computing the ground state solution of Bose-Einstein condensates by a normalized gradient flow. SIAM J Sci. Comput., 25:1674-1697, 2003.

[7] W. Bao, D. Jaksch, and P. Markowich. Numerical solution of the Gross-Pitaevskii equation for Bose-Einstein condensates. Journal of Computartional Physics, 187:318-342, 2003.

[8] T. Bartsch and Z.-Q. Wang. Note on ground states of nonlinear Schrödinger systems. J. Partial Differential Equations, 19(3):200-207, 2006.

[9] J. Bellazzini, M. Ghimenti, and S. Le Coz. Multi-solitary waves for the nonlinear KleinGordon equation. Comm. Partial Differential Equations, 39(8):1479-1522, 2014. 
[10] T. Cazenave. Semilinear Schrödinger equations. New York University - Courant Institute, New York, 2003.

[11] T. Cazenave and P.-L. Lions. Orbital stability of standing waves for some nonlinear Schrödinger equations. Comm. Math. Phys., 85(4):549-561, 1982.

[12] R. Côte and S. Le Coz. High-speed excited multi-solitons in nonlinear Schrödinger equations. J. Math. Pures Appl. (9), 96(2):135-166, 2011.

[13] R. Côte, Y. Martel, and F. Merle. Construction of multi-soliton solutions for the $L^{2}$ supercritical gKdV and NLS equations. Rev. Mat. Iberoam., 27(1):273-302, 2011.

[14] D. G. de Figueiredo and O. Lopes. Solitary waves for some nonlinear Schrödinger systems. Ann. Inst. H. Poincaré Anal. Non Linéaire, 25(1):149-161, 2008.

[15] J. Garnier and R. Marty. Effective pulse dynamics in optical fibers with polarization mode dispersion. Wave motion, 43(7):544-560, 2006.

[16] H. Hajaiej. Orbital stability of standing waves of some $\ell$-coupled nonlinear Schrödinger equations. Commun. Contemp. Math., 14(6):1250039, 10, 2012.

[17] I. Ianni and S. Le Coz. Multi-speed solitary wave solutions for nonlinear Schrödinger systems. J. Lond. Math. Soc. (2), 89(2):623-639, 2014.

[18] S. Le Coz, D. Li, and T.-P. Tsai. Fast-moving finite and infinite trains of solitons for nonlinear Schrödinger equations. Proc. Edinb. Math. Soc. (2), to appear.

[19] S. Le Coz and T.-P. Tsai. Infinite soliton and kink-soliton trains for nonlinear Schrödinger equations. Nonlinearity, 27(11):2689-2709, 2014.

[20] L. A. Maia, E. Montefusco, and B. Pellacci. Orbital stability property for coupled nonlinear Schrödinger equations. Adv. Nonlinear Stud., 10(3):681-705, 2010.

[21] S. V. Manakov. On the theory of two-dimensional stationary self-focusing of electromagnetic waves. Journal of Experimental and Theoretical Physics, 38, 1974.

[22] Y. Martel and F. Merle. Instability of solitons for the critical generalzed Korteweg-de-Vries equation. Geom. Funct. Anal., 11:74-123, 2001.

[23] Y. Martel and F. Merle. Multi solitary waves for nonlinear Schrödinger equations. Ann. Inst. H. Poincaré Anal. Non Linéaire, 23(6):849-864, 2006.

[24] Y. Martel, F. Merle, and T.-P. Tsai. Stability in $H^{1}$ of the sum of $K$ solitary waves for some nonlinear Schrödinger equations. Duke Math. J., 133(3):405-466, 2006.

[25] F. Merle. Construction of solutions with exactly $k$ blow-up points for the Schrödinger equation with critical nonlinearity. Comm. Math. Phys., 129(2):223-240, 1990.

[26] E. Montefusco, B. Pellacci, and M. Squassina. Energy convexity estimates for non-degenerate ground states of nonlinear 1D Schrödinger systems. Commun. Pure Appl. Anal., 9(4):867$884,2010$.

[27] N. V. Nguyen and Z.-Q. Wang. Existence and stability of a two-parameter family of solitary waves for a 2-coupled nonlinear Schrödinger system. preprint, 2015.

[28] M. Ohta. Stability of solitary waves for coupled nonlinear Schrödinger equations. Nonlinear Anal., 26(5):933-939, 1996.

[29] B. Sirakov. Least energy solitary waves for a system of nonlinear Schrödinger equations in $\mathbb{R}^{n}$. Comm. Math. Phys., 271(1):199-221, 2007.

[30] Z. Wang and S. Cui. Multi-speed solitary wave solutions for a coherently coupled nonlinear schrödinger system. Journal of Mathematical Physics, 56(2):1089-7658, 2015.

[31] M. I. Weinstein. Modulational stability of ground states of nonlinear Schrödinger equations. SIAM J. Math. Anal., 16:472-491, 1985.

(Fanny Delebecque and Stefan Le Coz) Institut de Mathématiques de Toulouse, UniverSité Paul Sabatier, 118 route de Narbonne, 31062 Toulouse Cedex 9, France

E-mail address, Fanny Delebecque: fanny.delebecque@math.univ-toulouse.fr

E-mail address, Stefan Le Coz: slecoz@math.univ-toulouse.fr

(Rada M. Weishäupl) Faculty of Mathematics, University of Vienna, Oskar-MorgensternPlatz 1, 1090 Vienna, Austria

E-mail address, Rada M. Weishäupl: rada.weishaeupl@univie.ac.at 